\title{
ON THE ORBIT SPACE OF A COMPACT LINEAR LIE GROUP WITH COMMUTATIVE CONNECTED COMPONENT
}

\author{
O. G. STYRT
}

\begin{abstract}
This paper is devoted to the study of topological quotients of compact linear Lie groups. More precisely, it investigates the question of when such a quotient is a topological or a smooth manifold.

The topological quotient of a finite linear group was studied by Mikhaulova in 1984. Here the connected component $G^{0}$ of the original Lie group $G$ is assumed to be a torus of positive dimension.

The main method used here is to consider the weight system corresponding to the decomposition of the representation of the commutative group $G^{0}$ into irreducible representations. In $\S 8$ an arbitrary linear group is reduced to a linear group of special type, namely, one with a 2-stable weight system (for the definition and properties of $q$-stable sets of vectors, where $q \in \mathbb{N}$, see $\S \S 1,4)$. The main results for a group with a 2 -stable weight system are stated in the Introduction (Theorems 1.3-1.8) and proved in $\S \S 6$ and 7 .
\end{abstract}

\section{INTRODUCTION}

Definition. A continuous map of smooth manifolds is called piecewise smooth if it takes any smooth submanifold to a finite union of smooth submanifolds.

In particular, every proper smooth map of smooth manifolds is piecewise smooth.

We consider a differentiable action of some compact Lie group $G$ on a smooth manifold $M$.

Definition. We say that the quotient of the action $G: M$ is diffeomorphic (piecewise diffeomorphic) to a smooth manifold $M^{\prime}$ if the topological quotient $M / G$ is homeomorphic to $M^{\prime}$ and the factorization map $M \rightarrow M^{\prime}$ is smooth (piecewise smooth).

Definition. We say that the quotient of the action $G: M$ is a smooth manifold if it is piecewise diffeomorphic to some smooth manifold.

Suppose that there is a faithful linear representation of a compact Lie group $G$ in a real vector space $V$. We are interested in the question of whether the quotient $V / G$ of this action is a topological manifold, and also whether it is a smooth manifold. For brevity, in what follows we will simply call a topological manifold a "manifold".

Notation. For an arbitrary linear representation $G: V$ and for a nonnegative integer $d$, when we write $\left(V \oplus \mathbb{R}^{d}\right) / G$, unless we stipulate otherwise, we will mean the quotient of the linear representation

$$
G: V \oplus \mathbb{R}^{d}, g: v+x \rightarrow g v+x, \quad g \in G, v \in V, x \in \mathbb{R}^{d} .
$$

Definition. A linear operator in a space over some field is called a reflection (a pseudoreflection) if its fixed point subspace has codimension 1 (2, respectively).

2000 Mathematics Subject Classification. Primary 22E45; Secondary 20C15, 22C05, 22E15.

Key words and phrases. Compact, linear group, Lie group, orbit space, smooth manifold. 
Mikhallova [1] obtained the following result in the case when the group $G$ is finite: if the quotient $V / G$ is diffeomorphic to the space $V$, then the group $G$ is generated by pseudoreflections, and if $G$ is generated by pseudoreflections, then the quotient $V / G$ is homeomorphic to $V$, and in particular, it is a manifold. We state a generalization of this result, which we prove in $\$ 2$.

Theorem 1.1. Suppose that the group $G$ is finite. If $\left(V \oplus \mathbb{R}^{d}\right) / G$ is a smooth manifold for some $d \geq 0$, then the group $G$ is generated by pseudoreflections, and if $G$ is generated by pseudoreflections, then $V / G$ is a manifold.

We denote by $G^{0}$ the connected component of the identity of the group $G$, and by $\mathfrak{g}$ its tangent algebra. In the space $V$ we can fix a $G$-invariant inner product. Then the group $G$ acts by orthogonal operators: $G \subset \mathbf{O}(V)$ and $G^{0} \subset \mathbf{S O}(V)$.

Let $G_{v}$ be the stabilizer of a vector $v \in V$, and let $\mathfrak{g}_{v}:=\operatorname{Lie} G_{v}=\{\xi \in \mathfrak{g}: \xi v=0\}$. The group $G_{v}$ leaves the vector $v$ and the orbit $G v$ invariant, and therefore it also leaves the tangent space $T_{v}(G v)=\mathfrak{g} v$ and the subspace $N_{v}:=(\mathfrak{g} v)^{\perp}$ invariant. We denote by $M_{v}$ the orthogonal complement in $N_{v}$ of the subspace $N_{v}^{G_{v}}$ of fixed vectors for the action $G_{v}: N_{v}$. Then $V=\mathfrak{g} v \oplus N_{v}^{G_{v}} \oplus M_{v}, G_{v} M_{v}=M_{v}$, and

$$
N_{v} / G_{v} \cong N_{v}^{G_{v}} \times\left(M_{v} / G_{v}\right)
$$

The space $M_{v}$ contains no nonzero vectors fixed by $G_{v}$; in particular, if it is nontrivial, then the group $G_{v}$ cannot act trivially on it.

For an arbitrary element $g \in G$ we introduce the notation

$$
\omega(g):=\operatorname{rk}(E-g)-\operatorname{rk}(E-\operatorname{Ad}(g)) .
$$

We set

$$
\Omega:=\{g \in G: \omega(g) \in\{0 ; 2\}\} \subset G .
$$

Let $v \in V$ be some vector with finite stabilizer. Then $\mathfrak{g}_{v}=0$ and for any $g \in G_{v}$ we have

$$
\operatorname{dim}\left((E-g) N_{v}\right)=\operatorname{dim}((E-g) V)-\operatorname{dim}((E-g)(\mathfrak{g} v)) .
$$

The map $\mathfrak{g} \rightarrow \mathfrak{g} v, \xi \rightarrow \xi v$ is a linear isomorphism of the spaces $\mathfrak{g}$ and $\mathfrak{g} v$, and if we use it to identify these spaces, then an element $g$ acts on $\mathfrak{g} v$ in the same way as the operator $\operatorname{Ad}(g)$ acts on $\mathfrak{g}$. In this case, $\operatorname{dim}((E-g)(\mathfrak{g} v))=\operatorname{rk}(E-\operatorname{Ad}(g))$ and, by (1.3), $\operatorname{dim}\left((E-g) N_{v}\right)=\omega(g)$. In particular, an element $g \in G_{v}$ belongs to $\Omega$ if and only if it acts on $N_{v}$ by a pseudoreflection or trivially.

If $W \subset V$ is a subspace, then the group $\mathbf{O}(W)$ embeds into the group $\mathbf{O}(V)$ as a subgroup of all orthogonal transformations of the space $V$ acting trivially on $W^{\perp}$. If $g \in \mathbf{O}(V)$, then $\mathbf{O}(g W)=g \mathbf{O}(W) g^{-1}$. Next, let $G[W]:=G \cap \mathbf{O}(W)$ be the Lie subgroup of all elements of $G$ acting trivially on $W^{\perp}$. The algebra $\mathfrak{g}[W]:=\operatorname{Lie} G[W] \subset \mathfrak{g}$ consists of all elements of the algebra $\mathfrak{g}$ that take $W^{\perp}$ to zero. For every $g \in G$ we have $G[g W]=g G[W] g^{-1}$ and $\mathfrak{g}[g W]=\operatorname{Ad}(g) \mathfrak{g}[W]$.

In this paper we consider the situation where the group $G^{0}$ is commutative; that is, it is a torus. Obviously, this property is preserved under passing to a quotient group.

Since $G^{0} \triangleleft G$, under the action of any $g \in G$ every $G^{0}$-invariant subspace goes to a $G^{0}$-invariant subspace, and an irreducible $G^{0}$-submodule goes to an irreducible submodule. Furthermore, the images of isomorphic $G^{0}$-submodules are isomorphic. The space $V$ can be decomposed into a direct sum of pairwise orthogonal irreducible $G^{0}$-invariant subspaces. Then the direct sums of isomorphic irreducible submodules (isotypic components) are also pairwise orthogonal, and the group $G$ permutes them.

Every irreducible representation of the commutative group $G^{0}$ has dimension 1 or 2 . Every two-dimensional representation has a complex structure according to which the 
group $G^{0}$ acts on the complex line by multiplication by numbers in the Lie group $\mathbb{T}:=$ $\{z \in \mathbb{C}:|z|=1\}$. The algebra Lie $\mathbb{T}$ can be identified with $\mathbb{R}$. Each two-dimensional irreducible representation of the group $G^{0}$ has a corresponding weight $\lambda: G^{0} \rightarrow \mathbb{T}$ which is a nontrivial homomorphism of Lie groups, as well as its differential, a nontrivial linear function $\lambda: \mathfrak{g} \rightarrow \mathbb{R}$. The space $\mathfrak{g}$ can be identified with $\mathfrak{g}^{*}$ by using an inner product invariant under $\operatorname{Ad}(G)$; therefore the linear function $\lambda$ can be understood as a vector in the space $\mathfrak{g}$. A weight $\lambda: G^{0} \rightarrow \mathbb{T}$ (a weight $\lambda \in \mathfrak{g}$ ) is determined up to pointwise inversion (change of sign, respectively), since on the complex line we can make the change of coordinates $z \rightarrow \bar{z}$. It is natural to associate the trivial weights $\lambda: G^{0} \rightarrow \mathbb{T}$ and the zero weights $\lambda=0 \in \mathfrak{g}$ with one-dimensional representations of the group $G^{0}$, since the group $G^{0}$ acts trivially on these representations.

Classes of isomorphic irreducible representations of the group $G^{0}$ are characterized by weights $\lambda \in \mathfrak{g}$ determined up to sign. The set $P$ of weights $\lambda$ corresponding to a decomposition of $V$ into a direct sum of irreducible components (taking their multiplicities into account) is independent of the decomposition and is invariant, up to sign (but taking account of the multiplicities), under the action of any operator $\operatorname{Ad}(g), g \in G$.

Let $V_{\lambda}(\lambda \in P)$ be the isotypic component corresponding to the irreducible representations with weights $\pm \lambda$. In particular, $V_{0}$ is the isotypic component corresponding to the one-dimensional irreducible representations. It is invariant under the action of $G$, and under the action of $G^{0}$, pointwise. We have $V_{\lambda_{1}}=V_{\lambda_{2}} \Leftrightarrow \lambda_{1}= \pm \lambda_{2}$. For a subset $Q \subset P$ we denote by $V_{Q}$ the sum of the isotypic components $V_{\lambda}$ over all $\lambda \in Q$.

Every isotypic component $V_{\lambda}, \lambda \neq 0$, has a complex structure, and an element $g \in G^{0}$ acts on it by multiplication by $\lambda(g)$, and $\xi \in \mathfrak{g}$ acts by multiplication by $i \lambda(\xi)$. Under the action of an element $g \in G$ the component $V_{\lambda}$ goes to the component $V_{\operatorname{Ad}(g) \lambda}$, and the subspace $V_{Q}$ goes to $V_{\operatorname{Ad}(g) Q}$. Furthermore, the component $V_{\lambda}(\lambda \neq 0)$ is invariant if and only if $\operatorname{Ad}(g) \lambda= \pm \lambda$. If in the last equality there is a "plus" sign ("minus" sign), then the restriction of $g$ to $V_{\lambda}$ commutes (anticommutes) with each $\xi \in \mathfrak{g}$ and therefore is linear (antilinear) over the field $\mathbb{C}$. Since the representation is faithful, the intersection of the kernels of all the weights $G^{0} \rightarrow \mathbb{T}$ in $P$ is trivial, while the intersection of the kernels of the corresponding weights $\lambda \in \mathfrak{g}$ is zero, and therefore $\mathfrak{g}$ is linearly generated by these weights, that is, $\langle P\rangle=\mathfrak{g}$.

For a $G^{0}$-invariant subspace $W \subset V$, the group $G[W] \cap G^{0}$ (algebra $\mathfrak{g}[W]$ ) is the intersection of the kernels of the weights $G^{0} \rightarrow \mathbb{T}(\mathfrak{g} \rightarrow \mathbb{R}$, respectively) corresponding to $W^{\perp}$.

A decomposition of a set of vectors of a finite-dimensional linear space into components is a representation of this set as a union of subsets whose linear hulls are linearly independent. If at least two of these linear hulls are nontrivial, then the decomposition is said to be proper. We say that a set is indecomposable if it does not admit any proper decompositions into components. Every set of vectors is uniquely decomposable into indecomposable components (up to distribution of the zero vector), and for any decomposition of it into components, each component is a union of some of its indecomposable components (again, up to the zero vector).

Definition. A finite set of vectors (taken with their multiplicities) in a finite-dimensional space is called $q$-stable $(q \geq 0)$ if its linear hull does not change when at most $q$ arbitrary vectors (counting multiplicities) are removed from it.

The addition and removal of zero vectors does not affect the $q$-stability of a set, nor is it affected by multiplying some vectors in the set by nonzero scalars. Clearly, the image of a $q$-stable set under a linear map is $q$-stable. In particular, if a finite set of linear functions on some space is $q$-stable, then the set of their restrictions to any subspace is also $q$-stable. 
It is easy to see that a set containing at most $q$ nonzero vectors is $q$-stable if and only if it does not contain nonzero vectors. Therefore any $q$-stable set with a $k$-dimensional linear hull $(k>0)$ contains more than $q$ nonzero vectors, and if $q$ of them are removed, then the linear hull must still be $k$-dimensional. Consequently, there cannot be less than $k+q$ nonzero vectors in such a set.

If a $q$-stable set is decomposed into components (not necessarily indecomposable ones), then each of the components is $q$-stable as the image of the original set under a linear map, namely the projection onto the corresponding direct summand (up to addition and removal of zeros).

We now state the results which we prove in what follows. First of all, the set of all nonzero weights in $P$ can be decomposed into a disjoint union of indecomposable components $\Lambda \subset P$. Let $L$ be the set of subspaces consisting of all the $V_{\Lambda}$ (where $\Lambda$ is an indecomposable component) and $V_{0}$. Then all the subspaces in $L$ are pairwise orthogonal, and $V$ is their direct sum.

The space $\mathfrak{g}$ is the direct sum of the linear hulls of all the indecomposable components $\Lambda \subset P$. The product of all the subgroups $G[W], W \in L$, is direct and contains $G^{0}$, since the direct sum of all the subspaces $\mathfrak{g}\left[V_{\Lambda}\right]$ is $\mathfrak{g}$. To a faithful representation $G\left[V_{\Lambda}\right]: V_{\Lambda}$ there corresponds an indecomposable set of weights.

In $\$ 5$ we shall prove the following theorem.

Theorem 1.2. Suppose that $P$ is a 2-stable set and $V / G$ is a smooth manifold. Then the group $G$ coincides with the direct product of all the subgroups $G[W], W \in L$. Furthermore, for any $W \in L$ there exists a number $d \geq 0$ such that $\left(W \oplus \mathbb{R}^{d}\right) / G[W]$ is a smooth manifold.

If the group $G$ coincides with the direct product of all the subgroups $G[W], W \in L$, and $W / G[W]$ is a manifold for each $W \in L$, then the quotient $V / G$ is also a manifold as the Cartesian product of the quotients $W / G[W], W \in L$. (This also holds without the requirement that $P$ be 2 -stable.)

If $P$ is a 2 -stable set, then each component $\Lambda$ is itself 2 -stable, and the set of weights corresponding to the action $G\left[V_{\Lambda}\right]: V_{\Lambda}$ is indecomposable, 2-stable, and contains no zeros.

In 88 the question of whether the quotient of an arbitrary representation of a Lie group of the type under consideration is a (smooth) manifold will be reduced to a similar question for a representation with a 2-stable set of weights.

In the case of a 2 -stable set $P$ we need to decompose $V$ into the direct sum of subspaces $W \in L$, verify whether $G$ coincides with the direct product of the subgroups $G[W], W \in L$ (if this is not the case, then $V / G$ is not a smooth manifold), and examine each action $G[W]: W, W \in L$ separately. For $W=V_{0}$ we can use Theorem 1.1, since the group $G[W]$ is finite.

We must clarify the situation of a 2 -stable indecomposable set $P$ containing no zeros. The following theorem describes this case under the additional condition $\operatorname{dim} G>1$.

Theorem 1.3. Suppose that the set of weights $P$ is indecomposable, 2-stable, and contains no zeros.

1. If $\operatorname{dim} G>1$ and $\left(V \oplus \mathbb{R}^{d}\right) / G$ is a smooth manifold for some $d \geq 0$, then the following four conditions hold:

1) the set $P$ is 2-stable and contains $m+2$ nonzero weights $(m=\operatorname{dim} G>0)$;

2 ) the space $V$ can be decomposed into a direct sum of pairwise orthogonal twodimensional $G^{0}$-invariant subspaces $W_{1}, \ldots, W_{m+2}$ permuted by the group $G$, and the latter leaves the subspace $W_{1} \oplus \cdots \oplus W_{m}$ and all the $W_{i}, i=1, \ldots$, $m+2, m>2$, invariant; 
3) there exists an element $g \in G$ leaving all the $W_{i}$ invariant such that $\operatorname{Ad}(g)=$ $-E$;

4) for any vector $v \in V$ with finite stabilizer we have $G_{v}=\left\langle G_{v} \cap \Omega\right\rangle$.

2. If $\operatorname{dim} G>0$ (it is not necessary that $\operatorname{dim} G>1$ ) and conditions 1) 4) hold, then $V / G$ is a manifold.

Theorem 1.4. If the representation $G: V$ satisfies conditions 1$)$ 3) of Theorem 1.3 and in addition the group $G$ leaves all the subspaces $W_{1}, \ldots, W_{m+2}$ invariant, then $V / G$ is a manifold.

Now suppose that the set $P$ is indecomposable, 2-stable, and contains no zeros, while the group $G$ is one-dimensional (or equivalently, $\operatorname{dim} G=1$, while $P$ contains at least 3 nonzero weights and no zero weights). The space $V$ has a complex structure, and $\operatorname{Ad}(G) \subset\{ \pm E\}$. An element $g \in G$ such that $\operatorname{Ad}(g)=E$ (such that $\operatorname{Ad}(g)=-E$ ) acts on $V$ linearly (antilinearly, respectively) over $\mathbb{C}$. In $\$ 3$ we shall prove that all the complex reflections of the group $G$ generate a finite subgroup $H \triangleleft G$, the quotient $V / H$ is diffeomorphic to $V$, and the group $G / H$ acts linearly on it. Obviously all the operators of the group $H$ are complex linear.

In $\$ 7$ we look at this case and prove Theorems 1.51 .8 for it.

Theorem 1.5. If $\left(V \oplus \mathbb{R}^{d}\right) / G$ is a smooth manifold for some $d$, then $\operatorname{dim}_{\mathbb{C}} V=3$, $\operatorname{Ad}(G)=\{ \pm E\}$, the group $G$ is generated by the set $\Omega$, and the representation $(G / H)$ : $(V / H)$ is reducible. If all these conditions hold, then the quotient $V / G$ is a manifold.

In the statements of the remaining three theorems we assume for convenience that $\operatorname{dim}_{\mathbb{C}} V=3$ and $\operatorname{Ad}(G)=\{ \pm E\}$.

Theorem 1.6. Suppose that the group $H$ is irreducible over the field $\mathbb{C}$ or $G$ is reducible over $\mathbb{R}$. If $\left(V \oplus \mathbb{R}^{d}\right) / G$ is a smooth manifold for some $d$, then $G=\langle\Omega\rangle$, and if $G=\langle\Omega\rangle$, then $V / G$ is a manifold.

Following [4], for arbitrary $p, q \in \mathbb{N}$ we denote by $G(p q, p, 3)$ the finite subgroup of $\mathbf{G L}_{3}(\mathbb{C})$ generated by all the operators of permutations of coordinates, as well as by all operators of the form $\operatorname{diag}\left(\varepsilon_{1}, 1,1\right)$ and $\operatorname{diag}\left(1, \varepsilon_{2}, \varepsilon_{2}^{-1}\right)$ with $\varepsilon_{1}^{q}=\varepsilon_{2}^{p q}=1$. The group $G(p q, p, 3)$ permutes the coordinate lines, and all its operators that result in an even permutation on the set of these lines form the subgroup $G^{\prime}(p q, p, 3)$ of index 2 . Let $G^{\prime}(q, 3) \subset \mathbf{G L}_{\mathbb{C}}(V), q \in \mathbb{N}$, be the one-dimensional Lie group generated by the group $G^{\prime}(q, 1,3)$ and all the scalar operators $\lambda E, \lambda \in \mathbb{T}$. Its normalizer contains the involutive operator $g_{(2,3)}:\left(z_{1}, z_{2}, z_{3}\right) \rightarrow\left(\overline{z_{1}}, \overline{z_{3}}, \overline{z_{2}}\right)$, which is antilinear over $\mathbb{C}$. We denote by $G(q, 3) \subset \mathbf{G L}_{\mathbb{R}}(V)$ the one-dimensional Lie group generated by $G^{\prime}(q, 3)$ and $g_{(2,3)}$.

Theorem 1.7. If the group $H$ is irreducible over the field $\mathbb{C}$ and does not coincide with any of the groups $G(3 q, 3,3), q \in \mathbb{N}$, then $V / G$ is a manifold.

Theorem 1.8. Suppose that the group $G$ is irreducible over $\mathbb{R}$ and $H$ is reducible over $\mathbb{C}$. If for some d the quotient $\left(V \oplus \mathbb{R}^{d}\right) / G$ is a smooth manifold, then $G=G(q, 3), q>1$, and if $G=G(q, 3), q>1$, then $V / G$ is a manifold.

When we assert that $V / G$ is a manifold, the question of whether this quotient is a smooth manifold remains open.

\section{Auxiliary facts}

Assertion 2.1. Suppose that $M_{1}, M_{2}, M_{3}$ are smooth manifolds, $f_{1}: M_{1} \rightarrow M_{2}$ and $f_{2}: M_{2} \rightarrow M_{3}$ are continuous maps, and the map $f_{1}$ is proper, smooth, and surjective. Then either both maps $f_{2}$ and $f:=f_{2} \circ f_{1}: M_{1} \rightarrow M_{3}$ are piecewise smooth or neither is. 
Proof. The image (inverse image) under the action of $f_{1}$ of any smooth submanifold of $M_{1}$ (of $M_{2}$ ) is a finite union of smooth submanifolds of $M_{2}$ (of $M_{1}$ ). Furthermore, $f\left(U_{1}\right)=f_{2}\left(f_{1}\left(U_{1}\right)\right)$ and $f_{2}\left(U_{2}\right)=f\left(f_{1}^{-1}\left(U_{2}\right)\right)$ for any subsets $U_{i} \subset M_{i}$.

Corollary 2.1. Suppose that we have a linear representation of a compact Lie group $G$ (not necessarily with a commutative connected component) in a vector space $V$. Suppose that for some normal subgroup $H \triangleleft G$ the quotient $V / H$ is diffeomorphic to a smooth manifold $M$. Then either both the quotients $M / G$ and $V / G$ are smooth manifolds or neither is.

We again consider a linear representation $G: V$ (as before, we do not require $G^{0}$ to be commutative). We denote the factorization map $V \rightarrow V / G$ by $\pi$.

Proposition 2.1. If $V / G$ is a smooth manifold, then there exists a connected $G$-invariant neighbourhood of zero $U$ in $V$ such that the quotient $U / G$ is piecewise diffeomorphic to an open ball of dimension $\operatorname{dim}(V / G)$.

Proof. The point $\pi(0) \in V / G$ has a neighbourhood that is diffeomorphic to a cell; that is, in $V$ there exists a $G$-invariant neighbourhood of zero $U$ such that the quotient $U / G$ is piecewise diffeomorphic to an open ball of dimension $\operatorname{dim}(V / G)$ and, in particular, is connected. In the neighbourhood $U$, every path-connected component is open. If $U^{0}$ is a path-connected component containing 0 , then the subsets $U^{0}$ and $U \backslash U^{0}$ are open and $G$-invariant. Their images under the factorization are open, disjoint, and their union is the connected quotient $U / G$. Consequently, the neighbourhood $U$ coincides with $U^{0}$ and is therefore connected.

Lemma 2.1. If some neighbourhood of the point $\pi(0)$ in $V / G$ is a (smooth) manifold, then $V / G$ is a (smooth) manifold.

Proof. In the space $V$ there exists a $G$-invariant neighbourhood $U$ of zero such that the quotient $U / G$ is a (smooth) manifold; the set $U$ contains some open ball $B$ centred at zero. This ball is $G$-invariant, and $B / G$ is a (smooth) manifold. Since there exists a diffeomorphism between $V$ and $B$ commuting with the action of $G$, the quotient $V / G$ is also a (smooth) manifold.

Theorem 2.1. Let $v \in V$ be some vector. The quotient $V / G$ is a manifold locally at the point $\pi(v)$ if and only if $N_{v} / G_{v}$ is a manifold. Furthermore, if $V / G$ is a smooth manifold, then $N_{v} / G_{v}$ is also a smooth manifold.

Proof. We set $H:=G_{v}$. By the slice theorem (2), Ch. II, $\left.\S \S 4,5\right)$, in the space $N_{v}$ there exists an $H$-invariant neighbourhood $U$ of the point $v$ such that the subset $G U$ is open in $V$ and is a homogeneous fibration $G_{H}^{*} U$, that is,

$$
U \cap g U=\varnothing \quad \forall g \in G \backslash H .
$$

If $V / G$ is a smooth manifold, then $(G U) / G$ is also a smooth manifold, since $G U$ is an open subset of $V$. The factorization map $G U \rightarrow(G U) / G$ restricted to $U$ gives the factorization of $U$ by $H$. Therefore, the quotient $U / H$ is also a smooth manifold. But then in the space $N_{v}$ there is an $H$-invariant neighbourhood of zero $U-v$ whose quotient by the action of $H$ is a smooth manifold: the map $U \rightarrow U-v, w \rightarrow w-v$ is a diffeomorphism commuting with the action of $H$.

The slice theorem also implies that the quotient $V / G$ locally at the point $\pi(v)$ is homeomorphic to the quotient $N_{v} / G_{v}$ locally at zero.

It remains to apply Lemma 2.1 to obtain both assertions of the theorem. 
Corollary 2.2. Let $v \in V$ be an arbitrary vector. If $\left(V \oplus \mathbb{R}^{d}\right) / G$ is a smooth manifold for some $d \geq 0$, then $\left(N_{v} \oplus \mathbb{R}^{d}\right) / G_{v}$ is also a smooth manifold.

Proof. Under the natural embedding of $V$ into $V \oplus \mathbb{R}^{d}$, on which the action (1.1) is defined, the orthogonal complement of the subspace $\mathfrak{g} v$ is exactly $N_{v} \oplus \mathbb{R}^{d}$. It remains to use Theorem 2.1

Corollary 2.3. If for some vector $v \in V$ the subspace $M_{v}$ is nontrivial and the group $G_{v}$ acts transitively on the unit sphere in it, then $V / G$ is not a manifold.

Proof. Clearly, the quotient of the space $M_{v}$ by the action of $G_{v}$ is homeomorphic to the half-line $\mathbb{R}_{\geq 0}$, and by (1.2) the quotient $N_{v} / G_{v}$ is homeomorphic to $N_{v}^{G_{v}} \times \mathbb{R}_{\geq 0}$, which is a closed half-space and thus is not a manifold. It follows from Theorem 2.1 that $V / G$ is not a manifold either.

In the space $V$ we can distinguish strata, that is, subsets of vectors with the stabilizer conjugate to a fixed subgroup of $G$. Altogether there are finitely many (nonempty) strata, each of them is $G$-invariant, and $V$ is their disjoint union. This means the quotient $V / G$ also splits into a disjoint union of the images of strata under the factorization, which we also call strata in $V / G$. For any vector $v \in V$, the stratum in $V$ containing $v$ is a smooth submanifold of $V$ of codimension $\operatorname{dim} M_{v}$. The image of this stratum under the factorization is also a smooth manifold and has codimension in $V / G$ equal to the dimension of the quotient $M_{v} / G_{v}$. In particular, since $\operatorname{dim}\left(M_{v} / G_{v}\right) \leq \operatorname{dim} M_{v}$, the codimension of a stratum in $V / G$ does not exceed the codimension of the corresponding stratum in $V$. The restriction of the map $\pi$ to any stratum is a locally trivial fibration. There is exactly one stratum of codimension 0 (that is, an open one) in the space $V$; it corresponds to the unique open stratum in $V / G$. We call these strata principal.

Lemma 2.2. If the quotient $V / G$ is a manifold, then it contains no strata of codimension 1 .

Proof. Suppose that there exists a stratum of codimension 1 , and let $v$ be a vector contained in it. Then $\operatorname{dim}\left(M_{v} / G_{v}\right)=1$; therefore the space $M_{v}$ is nontrivial, and the quotient of its unit sphere by the action of $G_{v}$ is discrete. By Corollary 2.3, the group $G_{v}$ cannot act transitively on the unit sphere in $M_{v}$. Thus, the quotient of this action consists of more than one point and, as it is discrete, it is not connected. But this can only happen if the space $M_{v}$ is one-dimensional and the group $G_{v}$ acts on it trivially, which in turn is impossible.

Assertion 2.2. In every $G^{0}$-invariant subspace $W \subset V$ there exists a vector $v$ such that $M_{v} \subset W^{\perp}$.

Proof. From the vectors in the space $W$ we choose a vector $v$ with minimal stabilizer, by inclusion. There exists a neighbourhood $U$ of the vector $v$ in $N_{v}$ satisfying (2.1). In particular, the stabilizer of any vector in $U$ is contained in $G_{v}$. Thus, the stabilizer of any vector in $W \cap U$ coincides with $G_{v}, v \in W \cap U \subset N_{v}^{G_{v}}, W \cap N_{v}=\langle W \cap U\rangle \subset N_{v}^{G_{v}}$, and $W=\mathfrak{g} v \oplus\left(W \cap N_{v}\right) \subset \mathfrak{g} v \oplus N_{v}^{G_{v}}=M_{v}^{\perp}$.

Lemma 2.3. Let $W \subset V$ be a nonzero $G^{0}$-invariant subspace such that the group $G[W]$ acts transitively on its unit sphere. Then $V / G$ is not a manifold.

Proof. We choose a vector $v \in W^{\perp}$ such that $M_{v} \subset W$. By hypothesis, there are no nonzero vectors in $W$ fixed by $G[W]$. In addition, $G[W] \subset G_{v}$, whence $W \cap N_{v}^{G_{v}}=0$. Furthermore, $M_{v} \subset W \subset N_{v}$; therefore $W=M_{v}$. Also, by hypothesis the action of the group $G_{v}$, which contains $G[W]$, is transitive on the unit sphere in the nonzero subspace $M_{v}$. It remains to apply Corollary 2.3 to obtain the required result. 
Corollary 2.4. If $V / G$ is a manifold, then there are no reflections in $G$ with respect to $G^{0}$-invariant hyperplanes.

Proof. If $g \in G$ is a reflection with respect to a $G^{0}$-invariant hyperplane $W^{\prime}$, then the operator $g \in G[W]$ acts on the $G^{0}$-invariant line $W:=\left(W^{\prime}\right)^{\perp}$ by multiplication by $(-1)$. Therefore the group $G[W]$ acts transitively on the unit sphere in the space $W$ (which consists of two points).

Corollary 2.5. If $V / G$ is a manifold, then for every two-dimensional $G^{0}$-invariant subspace $W$ the group $G[W]$ is finite.

Proof. The group $G[W]$ embeds into $\mathbf{O}(W) \cong \mathbf{O}_{2}$. If in addition $G[W]$ is infinite, then it contains the group $\mathbf{S O}_{2}$ of all rotations of the two-dimensional space $W$ and acts transitively on its unit sphere (the circle).

We denote by $U_{k}(k>0)$ the union of all the strata in $V$ whose images under the action of $\pi$ have codimension greater than $k$ in the quotient $V / G$. Then $U_{k} / G$ is the union of all the strata in $V / G$ of codimension greater than $k$, and $U_{k}$ is the union of some strata in $V$ which also all have codimension greater than $k$.

If $V / G$ is a smooth manifold, then there exists a connected $G$-invariant neighbourhood of zero $U \subset V$ such that the quotient $U / G$ is piecewise diffeomorphic to an open ball of dimension $\operatorname{dim}(V / G)$. In particular, this quotient is a connected smooth manifold, all its homotopy groups are trivial, and its subset $\left(U \cap U_{k}\right) / G$ is a union of finitely many smooth submanifolds of codimensions greater than $k$. Consequently, $\left(U \backslash U_{k}\right) / G$ is a connected topological space with trivial homotopy groups $\pi_{1}, \ldots, \pi_{k-1}$.

In the connected smooth manifold $U \subset V$, the subset $U \cap U_{k}$ is a union of finitely many smooth submanifolds of codimension greater than $k$. Therefore, the topological space $U \backslash U_{k}$ is nonempty and connected; hence the quotient $\left(U \backslash U_{k}\right) / G^{0}$ is also nonempty and connected. Furthermore, for any open ball $B \subset V$ the subset $B \backslash U_{k}$ in the space $V$ is nonempty, connected, and has trivial homotopy groups $\pi_{1}, \ldots, \pi_{k-1}$.

Theorem 2.2. If $V / G$ is a smooth manifold, then the quotient group $G / G^{0}$ is generated by cosets containing a representative that leaves some vector with a corresponding stratum in $V / G$ of codimension at most 2 invariant, that is, a vector in $V \backslash U_{2}$.

Proof. Consider a connected $G$-invariant neighbourhood of zero $U \subset V$ such that the quotient $U / G$ is piecewise diffeomorphic to an open ball of $\operatorname{dimension} \operatorname{dim}(V / G)$. As we have already determined, the topological space $\left(U \backslash U_{2}\right) / G^{0}$ is nonempty and connected, and its quotient $\left(U \backslash U_{2}\right) / G$ by the action of the finite group $G / G^{0}$ is simply connected. Therefore the quotient group $G / G^{0}$ is generated by elements that have a fixed point in $\left(U \backslash U_{2}\right) / G^{0}$. If an element of $G / G^{0}$ leaves some orbit of the action $G^{0}: U \backslash U_{2}$ invariant, then there exists a representative $g \in G$ of the corresponding coset that leaves some vector of this orbit invariant: $g v=v, v \in U \backslash U_{2}$.

We now prove Theorem 1.1 Suppose that $G$ is a finite group. Recall that it remains to prove the first assertion: if $\left(V \oplus \mathbb{R}^{d}\right) / G$ is a smooth manifold for some $d$, then $G$ is generated by pseudoreflections. It suffices to prove this for $d=0$ : the rank of the operator $E-g(g \in G)$ is the same on the spaces $V$ and $V \oplus \mathbb{R}^{d}$.

Thus, suppose that $V / G$ is a smooth manifold. According to Corollary 2.4 the group $G$ contains no reflections. If $v \in V$, then $\mathfrak{g} v=0, V=N_{v}=N_{v}^{G_{v}} \oplus M_{v}=V^{G_{v}} \oplus M_{v}$, and the codimension of the stratum in $V / G$ corresponding to $v$ is $\operatorname{dim}\left(M_{v} / G_{v}\right)=\operatorname{dim} M_{v}$, since $\left|G_{v}\right|<\infty$.

By Theorem 2.2, the group $G$ is generated by elements $g$ that leave invariant some vector $v$ that has a corresponding stratum in $V / G$ of codimension at most 2 , that is, such 
that $\operatorname{dim} M_{v} \leq 2$. An operator $g \in G_{v}$ acts trivially on the subspace $V^{G_{v}}$; therefore, $\operatorname{dim}((E-g) V) \leq \operatorname{dim} M_{v} \leq 2$. Thus, $g$ is the identity operator or a pseudoreflection (there are no reflections in the group $G$ ).

Thus Theorem 1.1 is proved.

Corollary 2.6. Suppose that the stabilizer $G_{v}$ of some vector $v \in V$ is finite.

1) If $\left(V \oplus \mathbb{R}^{d}\right) / G$ is a smooth manifold for some $d$, then $G_{v}=\left\langle G_{v} \cap \Omega\right\rangle$, and if $G_{v}=\left\langle G_{v} \cap \Omega\right\rangle$, then locally at the point $\pi(v)$ the quotient $V / G$ is a manifold.

2) If $G_{v}=\left\langle G_{v} \cap \Omega\right\rangle$ and the vector $v$ lies on the unit sphere $S \subset V$, then locally at the point $\pi(v)$ the quotient $S / G$ is a manifold.

Proof. The set of all elements of the group $G_{v}$ acting on $N_{v}$ by a pseudoreflection or trivially coincides with $G_{v} \cap \Omega$.

If $v \in S$, then the orthogonal complement of the subspace $\mathfrak{g} v$ in the tangent space $T_{v}(S)=\langle v\rangle^{\perp}$ is $N_{v}(S):=N_{v} \cap\left(T_{v} S\right)=N_{v} \cap\langle v\rangle^{\perp}$. Clearly, $N_{v}=N_{v}(S) \oplus\langle v\rangle$. Each element in $G_{v} \cap \Omega$ acts on the line $\langle v\rangle$ trivially, and on $N_{v}(S)$, as on $N_{v}$, by a pseudoreflection or trivially.

It remains to use Theorems 2.1 and 1.1, as well as Corollary 2.2.

Theorem 2.3. Suppose that an orbit in general position of the representation $G: V$ has nontrivial homotopy group $\pi_{k}(k>0)$, and in the quotient $V / G$ any stratum distinct from the principal stratum has codimension greater than $k+2$. Then $V / G$ is not a smooth manifold.

Proof. By hypothesis the principal stratum in $V$ coincides with $V \backslash U_{k+2}$.

Suppose that $V / G$ is a smooth manifold. Then there exists a connected $G$-invariant neighbourhood of zero $U \subset V$ such that the quotient $U / G$ is piecewise diffeomorphic to an open ball of dimension $\operatorname{dim}(V / G)$. The subset $U^{\prime}:=U \backslash U_{k+2}$ is open, connected, $G$-invariant, and is contained in the principal stratum of the space $V$. The subset $U^{\prime} / G$ in $V / G$ is connected, and its homotopy groups $\pi_{1}, \ldots, \pi_{k+1}$ are trivial. The map $\pi$ gives a locally trivial fibration when restricted to the principal stratum, and therefore also when restricted to $U^{\prime}$.

The neighbourhood $U$ of zero contains an open ball $B$ of some radius $\delta>0$ with centre at zero. The set $U^{\prime} \cap B=B \backslash U_{k+2}$ is nonempty, $G$-invariant, connected, and has trivial homotopy groups $\pi_{1}, \ldots, \pi_{k+1}$. Let $o \in U^{\prime} \cap B$ be some vector. It belongs to the principal stratum, and by hypothesis the group $\pi_{k}(G o)$ is nontrivial. The fibration $U^{\prime} \rightarrow U^{\prime} / G$ has fibre Go. We consider the segment of the exact homotopy sequence

$$
\pi_{k+1}\left(U^{\prime} / G\right) \stackrel{\rho_{k+1}}{\longrightarrow} \pi_{k}(G o) \stackrel{\tau_{k}}{\longrightarrow} \pi_{k}\left(U^{\prime}\right) .
$$

Since the group $\pi_{k+1}\left(U^{\prime} / G\right)$ is trivial, the homomorphism $\tau_{k}$ is an embedding. Next, $G o \subset U^{\prime} \cap B$; consequently, for any element $\gamma \in \pi_{k}(G o)$, a $k$-dimensional spheroid in $U^{\prime}$ that is entirely contained in $U^{\prime} \cap B$ corresponds to the element $\tau_{k}(\gamma) \in \pi_{k}\left(U^{\prime}\right)$. This spheroid contracts to a point in the subset $U^{\prime} \cap B$ with trivial group $\pi_{k}$, and therefore also in the entire set $U^{\prime}$, whence the element $\tau_{k}(\gamma)$ is trivial. Since $\gamma$ is arbitrary, the homomorphism $\tau_{k}$ is trivial, but at the same time it is an embedding and the group $\pi_{k}(G o)$ is nontrivial. We have obtained a contradiction.

Corollary 2.7. Under the hypotheses of Theorem 2.3 , the quotient $\left(V \oplus \mathbb{R}^{d}\right) / G$ is not a smooth manifold for any $d$.

Proof. It suffices to prove that for $d \geq 0$ the representation (1.1) satisfies the hypotheses of Theorem 2.3 .

The quotient of this action is homeomorphic to $V / G \times \mathbb{R}^{d}$, the strata in it are exactly the Cartesian products of the strata in $V / G$ by $\mathbb{R}^{d}$, and their codimensions are equal to 
the codimensions of the corresponding strata in $V / G$. Finally, an orbit in general position for the action $G: V$ is also an orbit in general position for the representation (1.1).

Lemma 2.4. Suppose that $V$ is a direct sum of two $G$-invariant subspaces $V^{\prime}$ and $V^{\prime \prime}$, and the quotient of the action of $G$ on the unit sphere $S \subset V$ is a manifold. Furthermore, suppose that the quotient of the unit sphere in $V^{\prime \prime}$ by the action of $G$, that is, $\left(S \cap V^{\prime \prime}\right) / G$, is homeomorphic to a closed ball (of some dimension), while all the homotopy groups of the quotient $\left(V^{\prime} \backslash\{0\}\right) / G$ are trivial (this also means that this quotient is connected). Then $V / G$ is a manifold.

Proof. We denote by $\theta_{t}(t \in \mathbb{R}, 0 \leq t \leq 1)$ the linear map in $V$ that acts on $V^{\prime}$ as the identity map, and acts on $V^{\prime \prime}$ by multiplication by $t$. Then $\theta:[0 ; 1] \times V \rightarrow V$ is a continuous map, $\theta_{1}=E$, and $\theta_{0}(V)=V^{\prime}$. It follows from the equalities $G V^{\prime}=V^{\prime}$ and $G V^{\prime \prime}=V^{\prime \prime}$ that all the $\theta_{t}$ commute with all operators in $G$, and the set $U:=V \backslash V^{\prime \prime}$ is invariant under all the maps $\theta_{t}$ and all $g \in G$, and under the action of $\theta_{0}$, this set goes to $U \cap V^{\prime}$. Therefore, in fact, we have maps $\widetilde{\theta}_{t}: U / G \rightarrow U / G$ such that $\widetilde{\theta}:[0 ; 1] \times U / G \rightarrow U / G$ is a continuous map, $\widetilde{\theta}_{1}$ is the identity transformation in $U / G$, and the image of $\widetilde{\theta}_{0}$ is contained in the subset $\left(U \cap V^{\prime}\right) / G$, where, in turn, all the $\widetilde{\theta}_{t}$ are identity maps. Hence the quotient $U / G$ is homotopy equivalent to the quotient

$$
\left(U \cap V^{\prime}\right) / G=\left(V^{\prime} \backslash\{0\}\right) / G,
$$

all of whose homotopy groups are trivial by hypothesis. Therefore all the homotopy groups of $U / G$ are also trivial.

If we remove the subset $\left(S \cap V^{\prime \prime}\right) / G$, which is homeomorphic to a closed ball from the quotient $S / G$, then the remaining subset after taking the Cartesian product with $\mathbb{R}_{>0}$ gives a topological space homeomorphic to $U / G$. Therefore, all the homotopy groups of the remaining topological space are trivial, like those of $U / G$. Thus, after a subset homeomorphic to a closed ball is removed from the manifold $S / G$, a topological space all of whose homotopy groups are trivial is left. Consequently, the quotient $S / G$ is homeomorphic to a sphere, and the cone $V / G$ over this quotient to a vector space.

Throughout what follows we assume that the group $G^{0}$ is commutative.

In each isotypic component $V_{\lambda}(\lambda \in P)$ of the representation $G^{0}: V$ the stabilizer of any nonzero vector in the group $G^{0}$ coincides with the kernel of the homomorphism $\lambda: G^{0} \rightarrow \mathbb{T}$. If a vector $v \in V$ has nonzero projections onto each $V_{\lambda}$, then its stabilizer in the group $G^{0}$ coincides with the intersection of the kernels of all weights $\lambda: G^{0} \rightarrow \mathbb{T}$, which is trivial; hence $\left|G_{v}\right|<\infty$. Thus, a typical vector in the space $V$ has finite stabilizer in $G$, a typical orbit has $\operatorname{dimension} \operatorname{dim} G$, and $\operatorname{dim}(V / G)=\operatorname{dim} V-\operatorname{dim} G$.

As the group $G^{0}$ is commutative, thus $\operatorname{Ad}\left(G^{0}\right)=\{E\}$. In particular, for any $v \in V$, the group $G_{v} \cap G^{0}$ acts trivially on $\mathfrak{g} v$.

For an arbitrary subset $Q \subset P$ we consider the Lie group $\mathbb{T}^{|Q|}$ in which each element $z$ is determined by its coordinates $z_{\lambda} \in \mathbb{T}(\lambda \in Q)$. In the algebra Lie $\mathbb{T}^{|Q|}=\mathbb{R}^{|Q|}$, each element $x$ is determined by its coordinates $x_{\lambda} \in \mathbb{R}(\lambda \in Q)$.

We construct a homomorphism of Lie groups $\varphi_{Q}: G^{0} \rightarrow \mathbb{T}^{|Q|}$ by setting $\left(\varphi_{Q}(g)\right)_{\lambda}:=$ $\lambda(g)$. This homomorphism has the differential $d \varphi_{Q}: \mathfrak{g} \rightarrow \mathbb{R}^{|Q|}$ given by $\left(d \varphi_{Q}(\xi)\right)_{\lambda}=\lambda(\xi)$.

Proposition 2.2. If $V / G$ is a manifold, then the set $P \subset \mathfrak{g}$ is 1-stable.

Proof. We need to prove that when we remove any weight $\lambda$ from $P$ (accounting for multiplicity), the kernels of the remaining set of weights have trivial intersection, or equivalently, have linear hull $\mathfrak{g}$. If $\lambda=0$, then there is nothing to prove. If $\lambda$ is a nonzero weight, then a two-dimensional irreducible subrepresentation $W \subset V$ of the group $G^{0}$ corresponds to it. By Corollary 2.5, the group $G[W]$ is finite, and the intersection of the 
kernels of all the weights remaining after removing $\lambda$ from $P$ (accounting for multiplicity) is $\mathfrak{g}[W]=\operatorname{Lie} G[W]=0$, as required.

Lemma 2.5. Let $W \subset V$ be some $G^{0}$-invariant subspace, and suppose that the group $G[W]$ has dimension $k>0$. If in addition $V / G$ is a manifold, then $\operatorname{dim} W \geq 2(k+1)$.

Proof. The linear representation $G[W]: V$ is faithful; therefore the corresponding set $P^{\prime}$ of weights $\mathfrak{g}[W] \rightarrow \mathbb{R}$ linearly generates a $k$-dimensional space dual to $\mathfrak{g}[W]$. This set is 1-stable, since it consists exactly of the restrictions of all weights of the 1-stable set $P$ to the subspace $\mathfrak{g}[W] \subset \mathfrak{g}$. Since $k>0$, it follows that $P^{\prime}$ contains at least $k+1$ nonzero weights. Furthermore, the group $G[W]$ acts trivially on the subspace $W^{\perp}$; hence all the nonzero weights in $P^{\prime}$ correspond (accounting for multiplicities) to the two-dimensional irreducible subrepresentations in the representation $(G[W])^{0}: W$. Therefore, $\operatorname{dim} W \geq 2(k+1)$.

For the remainder of this section, we will assume that $\left(V \oplus \mathbb{R}^{d}\right) / G$ is a smooth manifold for some $d$.

Theorem 2.4. The quotient group $G / G^{0}$ is generated by cosets containing a representative of some finite group $G_{v}, v \in V$.

Proof. We claim that if a vector $v \in V$ has a corresponding stratum in $V / G$ of codimension at most 2 , then $\left|G_{v}\right|<\infty$. Indeed, the group $G_{v} \cap G^{0}$ acts trivially on the subspaces $N_{v}^{G_{v}}$ and $\mathfrak{g} v$, whence $G_{v} \cap G^{0} \subset G\left[M_{v}\right]$. If $k:=\operatorname{dim} G_{v}>0$, then $\operatorname{dim} G\left[M_{v}\right] \geq \operatorname{dim}\left(G_{v} \cap G^{0}\right)=k$; hence, according to Lemma 2.5. $\operatorname{dim} M_{v} \geq 2(k+1)$. Therefore the codimension of the corresponding stratum in $V / G$ is equal to

$$
\operatorname{dim}\left(M_{v} / G_{v}\right)=\operatorname{dim} M_{v}-\operatorname{dim} G_{v} \geq 2(k+1)-k=k+2>2,
$$

which contradicts our assumption. Consequently, the vector $v$ has finite stabilizer.

We can now use Theorem 2.2 and obtain the required assertion.

Corollary 2.8. The quotient group $G / G^{0}$ is generated by cosets that intersect $\Omega$. Equivalently, the group $G$ is generated by the set $G^{0} \cup \Omega$.

Proof. The proof immediately follows from Theorem 2.4 and Corollary 2.6.

Corollary 2.9. The group $\operatorname{Ad}(G)$ is generated by the operators $\operatorname{Ad}(g)$ over all $g \in \Omega$.

Theorem 2.5. If $\operatorname{dim} G>0$, then in the quotient $V / G$ there exists a stratum of positive codimension not greater than 3 .

Proof. It is sufficient to prove that an orbit in general position for the action $G: V$ is not simply connected; after that, setting $k:=1$ we can apply Corollary 2.7.

If $H \subset G$ is a stabilizer in general position, then $|H|<\infty$, and the orbit in general position is homeomorphic to the manifold $G / H$ of the left cosets of $H$ in $G$. The map $G \rightarrow G / H, g \rightarrow g H$ is a covering with finite fibre $H$, and the covering space is not simply connected, since $G^{0}$ is a torus of positive dimension. Therefore the base $G / H$ is not simply connected either.

\section{BASIC EXAMPLES}

3.1. Finite groups generated by complex reflections. Suppose that a space $V$ has a complex structure, that is, $\operatorname{dim}_{\mathbb{C}} V=n$, and there is a compact Lie group $G$ acting on it, consisting of $\mathbb{R}$-linear operators each of which is linear or antilinear over $\mathbb{C}$.

Lemma 3.1. Suppose that a Lie group $G$ acts in some complex space by $\mathbb{C}$-linear and $\mathbb{C}$-antilinear operators. Then for any $G$-invariant complex subspace there exists a complementary G-invariant complex subspace. 
Proof. By hypothesis the group $G$ normalizes the group

$$
\Gamma:=\{ \pm E ; \pm I\}
$$

generated by the operator $I$ of multiplication by $i$; therefore $G \Gamma$ is a compact Lie group, and any real linear representation of it is completely reducible. It remains to observe that the $(G \Gamma)$-invariant real subspaces are exactly the $G$-invariant complex ones.

Let $H \triangleleft G$ be some finite normal subgroup generated by (complex) reflections of the space $V$.

Theorem 3.1. The quotient $V / H$ is diffeomorphic to the space $V$, and every operator in $G$ that is linear (antilinear) over $\mathbb{C}$ acts on $V / H$ linearly (antilinearly) over $\mathbb{C}$.

Proof. The group $G$ acts on the linear space $\mathbb{C}[V]$ by the formula

$$
g f(v):= \begin{cases}f\left(g^{-1} v\right), & g \in \mathbf{G L}_{\mathbb{C}}(V) ; \\ \overline{f\left(g^{-1} v\right),}, & g \notin \mathbf{G L}_{\mathbb{C}}(V) .\end{cases}
$$

A $\mathbb{C}$-linear ( $\mathbb{C}$-antilinear) operator in the group $G$ acts on $\mathbb{C}[V]$ linearly (antilinearly) over $\mathbb{C}$. Furthermore, $g\left(f_{1} f_{2}\right)=\left(g f_{1}\right)\left(g f_{2}\right)$ for $g \in G, f_{i} \in \mathbb{C}[V]$.

The first assertion of the theorem follows from the Shephard-Todd-Chevalley Theorem: the factorization map can be given by the formula

$$
\pi_{H}: V \rightarrow V / H, \quad v \rightarrow\left(f_{1}(v), \ldots, f_{n}(v)\right),
$$

where $\left\{f_{1}, \ldots, f_{n}\right\}$ is any system of algebraically independent homogeneous generators of the algebra of invariants $B:=\mathbb{C}[V]^{H}$. Since $H \triangleleft G$, we have $G B=B$. Let $B_{k} \subset B$ be the subspace of homogeneous invariants of degree $k$.

In each $B_{k}(k>0)$ we can distinguish the subspace $C_{k}$ spanned by all the $B_{i} B_{k-i}$ $(0<i<k)$, that is, the subspace generated by all possible products of polynomials in $B$ of degree less than $k$, where the products themselves are homogeneous and have degree $k$. The finite-dimensional complex space $B_{k}$ and its complex subspace $C_{k}$ are invariant under $G$; therefore there exists a $G$-invariant complex subspace $C_{k}^{\prime} \subset B_{k}$ such that $B_{k}=C_{k} \oplus C_{k}^{\prime}$. By choosing a basis in each $C_{k}^{\prime}$ we obtain a system $\left(f_{1}, \ldots, f_{n}\right)$ of algebraically independent homogeneous generators of the algebra $B$. We define the factorization map by the formula $\pi_{H}(v):=\left(f_{1}(v), \ldots, f_{n}(v)\right)$. Then $\pi_{H}(0)=0$, since the $f_{i}$ are polynomials without a free term.

For any $g \in G$ we have $g C_{k}^{\prime}=C_{k}^{\prime}$. If $g$ is a linear (antilinear) operator in $V$, then it acts on $V / H$ linearly (antilinearly) over $\mathbb{C}$, since each number $f_{i}(g v)\left(\overline{f_{i}(g v)}\right)$ can be complex-linearly expressed in terms of the numbers $f_{j}(v)$ such that $\operatorname{deg} f_{j}=\operatorname{deg} f_{i}$.

Corollary 3.1. For any $d \geq 0$, the quotient $\left(V \oplus \mathbb{R}^{d}\right) / H$ is diffeomorphic to the space $V \oplus \mathbb{R}^{d}$, and every $\mathbb{C}$-linear ( $\mathbb{C}$-antilinear) operator in $G$ acts on the direct summand $\mathbb{R}^{d}$ of this quotient trivially, and on the direct summand $V$ linearly (antilinearly) over $\mathbb{C}$.

As can be seen from the proof of Theorem 3.1, in the space $V / H$ the linear hulls of all the basis vectors corresponding to homogeneous generators of any fixed degree are $G$-invariant. In particular, if the representation $G:(V / H)$ is irreducible, then the degrees of all the homogeneous generators are the same.

Thus, we have obtained a representation of the Lie group $G / H$ in the space $V / H$ that is linear (over $\mathbb{R}$ ). We now prove that it is faithful, that is, that any element $g \in G$ acting on $V / H$ trivially belongs to $H$. Indeed, for any $v \in V$ we have $g v \in H v$ and $h^{-1} g v=v$ for some $h$. We see that $V$ is the union of linear subspaces (over $\mathbb{R}$ ) of fixed points of operators $h^{-1} g, h \in H$ that are also linear over $\mathbb{R}$. Since $|H|<\infty$, for some $h \in H$ this 
subspace coincides with $V$, whence $g=h$. The group $G / H$ can be identified with its image in $\mathbf{G L}_{\mathbb{R}}(V / H)$ under the faithful representation thus obtained.

Suppose that in the group $G / H$ there is some finite subgroup $G^{\prime} / H$ generated by reflections, where $H \subset G^{\prime} \subset G$. In particular, $G^{\prime} / H$ consists only of $\mathbb{C}$-linear operators in $V / H$. Therefore, all elements in $G^{\prime}$ act on $V$ complex-linearly. Since the groups $H$ and $G^{\prime} / H$ are finite, the group $G^{\prime}$ is also finite. Since $G^{\prime} / H$ is generated by reflections, we have

$$
V / G^{\prime} \cong(V / H) /\left(G^{\prime} / H\right) \cong V / H \cong V ;
$$

hence the group $G^{\prime}$ is generated by reflections of the space $V$. In particular, if $H$ is generated by all reflections of the group $G$, then $G / H$ contains no reflections, for otherwise $G / H$ would contain a nontrivial finite subgroup $G^{\prime} / H$ generated by reflections for $H \varsubsetneqq G^{\prime} \subset G$, and the group $G^{\prime}$ would be generated by reflections, which is impossible.

Suppose that $V_{0}=0$ and the subgroup $G^{0} \subset G$ is central, that is, $\operatorname{Ad}(G)=\{E\}$. Then $G$ leaves all the isotypic components $V_{\lambda}(\lambda \in P)$ of the representation $G^{0}: V$ invariant and acts on each of them complex-linearly. Thus, a complex structure is also introduced on the space $V$ as the direct sum of the $V_{\lambda}$, and the group $G$ acts linearly on the complex space $V$. For a one-dimensional Lie group $G$ the condition $V_{0}=0$ is equivalent to requiring that the stabilizer of any nonzero vector in $V$ is finite (which is the same as requiring that it does not contain $G^{0}$ ).

Proposition 3.1. If $\operatorname{dim} G=1, V_{0}=0, \operatorname{Ad}(G)=\{E\}, \operatorname{dim}_{\mathbb{C}} V>1$, then the subgroup $H \triangleleft G$ generated by all the (complex) reflections of the group $G$ is finite.

Proof. Since $G V_{\lambda}=V_{\lambda}$ for all $\lambda$, any reflection in $G$ acts trivially on all the $V_{\lambda^{\prime}}$ apart from one $V_{\lambda}$ on which it acts by a complex reflection, that is, it belongs to the group $G\left[V_{\lambda}\right]$. For each $\lambda$, let $H_{\lambda}$ denote the group generated by all reflections in $G\left[V_{\lambda}\right]$. Then $H$ is the direct product of all the $H_{\lambda}$.

If there exist at least two isotypic components, then each $H_{\lambda}$ acts trivially on each $V_{\lambda^{\prime}}$ for $\lambda^{\prime} \neq \pm \lambda$; therefore $H_{\lambda} \cap G^{0}$ is contained in the kernel of the nontrivial weight $\lambda^{\prime}: G^{0} \rightarrow \mathbb{T}$. This kernel is finite; hence the group $H_{\lambda}$ is also finite. Therefore the group $H$ is also finite as the direct product of all the $H_{\lambda}$.

If there is only one isotypic component $V_{\lambda}=V$, then $G^{0}$ acts on the space $V$ by scalar operators, and for an arbitrary reflection $g \in G$ the operator $g^{\left|G / G^{0}\right|} \in G^{0}$ is a reflection or the identity operator. Since a scalar operator cannot be a reflection in a space of dimension greater than 1 , we have $g^{\left|G / G^{0}\right|}=E$ and

$$
(\operatorname{det} g)^{\left|G / G^{0}\right|}=1
$$

(the determinant is complex). All the elements $g \in G$ satisfying (3.2) form a subgroup, which contains all the reflections in $G$ and therefore also the subgroup $H$. But any operator $g \in H \cap G^{0}$ is scalar, has the form $\lambda E$, and the number $\lambda$ can take only finitely many values in view of (3.2). Consequently, $\left|H \cap G^{0}\right|<\infty$ and $|H|<\infty$.

The quotient $\left(V \oplus \mathbb{R}^{d}\right) / H(d \geq 0)$ is diffeomorphic to the space $V \oplus \mathbb{R}^{d}$, the one-dimensional group $G / H$ acts on its direct summand $V$ by complex linear operators among which there are no reflections, and it acts trivially on the direct summand $\mathbb{R}^{d}$. The connected component of the identity element of the group $G$ is contained in its centre; therefore the same property is also enjoyed by its homomorphic image $G / H$. Finally, for the action $(G / H):(V / H)$ the stabilizer of any nonzero vector is finite. Indeed, if not, the group $(G / H)^{0}=\left(G^{0} H\right) / H$ would leave the nonzero vector $v^{\prime}=\pi_{H}(v) \in V / H$ invariant, where $v \in V, v \neq 0$; then $G^{0} v \subset H v$ and, since $G^{0}$ is connected and $H$ is finite, we would have $G^{0} v=\{v\}$. This contradicts the condition $V_{0}=0$. 
Theorem 3.2. If $\operatorname{dim} G=1, \operatorname{Ad}(G)=\{E\}, V_{0}=0$, and the set $P$ contains at least three nonzero weights, then the quotient $\left(V \oplus \mathbb{R}^{d}\right) / G$ is not a smooth manifold for any $d$.

Proof. Suppose that $\left(V \oplus \mathbb{R}^{d}\right) / G$ is a smooth manifold, where $d \geq 0$.

The group $G$ acts linearly on $V$ as on a complex space. By hypothesis, $\operatorname{dim}_{\mathbb{C}} V>2$. Therefore all the elements of $G$ acting on $V$ by complex reflections generate a finite subgroup $H \triangleleft G$. As we already know, the quotient $\left(V \oplus \mathbb{R}^{d}\right) / H$ is diffeomorphic to the space $V \oplus \mathbb{R}^{d}$. The group $G / H$ acts canonically on the latter, and the quotient of this action is a smooth manifold, since $\left(V \oplus \mathbb{R}^{d}\right) / G$ is a smooth manifold.

Thus everything is reduced to the case where $\left(V \oplus \mathbb{R}^{d}\right) / G$ is a smooth manifold, the group $G$ acts on $V$ complex-linearly without complex reflections, and, as before, $V_{0}=0$ and $\operatorname{dim}_{\mathbb{C}} V>2$. By Theorem 2.5 there is a stratum in $V / G$, distinct from the principal one, with codimension at most 3 . In such a stratum we consider a point $\pi(v) \in V / G$, where $v \in V$. If $\left|G_{v}\right|=\infty$, then $v=0$; therefore this stratum in $V / G$ consists of only the point $\pi(0)$ and has codimension $\operatorname{dim}(V / G)=2 \operatorname{dim}_{\mathbb{C}} V-1 \geq 5$. Therefore, $\left|G_{v}\right|<\infty$ and the codimension of the stratum in $V / G$ is equal to $\operatorname{dim} M_{v}$, so $0<\operatorname{dim} M_{v}<4$. Since $\operatorname{Ad}(G)=\{E\}$, the complex subspace $V^{G_{v}}$ coincides with $N_{v}^{G_{v}} \oplus \mathfrak{g} v$ and has complex codimension $\frac{1}{2} \operatorname{dim} M_{v}<\frac{4}{2}=2$. At the same time the group $G_{v}$ is nontrivial, since the corresponding stratum is nonprincipal and contains no complex reflections. We have arrived at a contradiction.

3.2. The action of the torus on a complex space. Suppose that a space $V$ again has a complex structure, $\operatorname{dim}_{\mathbb{C}} V=n \in \mathbb{N}$, and that it decomposes into a direct sum of pairwise orthogonal one-dimensional complex subspaces $W_{1}, \ldots, W_{n}$.

Theorem 3.3. Suppose that an n-dimensional compact Lie group $G \subset \mathbf{O}(V)$ acts in the space $V$ permuting the subspaces $W_{i}$, and if $n>2$, then $G W_{i}=W_{i}$ for all $i$. Then the quotient of the unit sphere $S \subset V$ by the action of the group $G$ is homeomorphic to a closed $(n-1)$-dimensional ball; in particular, all the homotopy groups of the quotients $S / G$ and $(V \backslash\{0\}) / G$ are trivial.

Proof. The Lie group $G^{0}$ leaves all the subspaces $W_{i}$ invariant and acts on each of them complex-linearly. Hence $G^{0}$ embeds into the connected Lie group $\mathbb{T}^{n}$, which means that this embedding is an isomorphism, so that $V / G^{0} \cong \mathbb{R}_{\geq 0}^{n}$. Let $g \in G$ be an arbitrary element. If it leaves all the subspaces $W_{i}$ invariant, then it acts on the quotient $V / G^{0}$ trivially, since it belongs to $\mathbf{O}(V)$. If not, we have $n=2$, the element $g$ takes $W_{1}$ and $W_{2}$ to each other and acts on the quotient $V / G^{0} \cong \mathbb{R}_{\geq 0}^{2}$ by transposing the two coordinates with respect to $\mathbb{R}_{\geq 0}$. In either case, the quotient $V / G$ is homeomorphic to a closed $n$-dimensional half-space and has $\pi(0)$ as a boundary point, while the quotient $S / G$ is homeomorphic to a closed $(n-1)$-dimensional ball: this is either a simplex on the unit sphere in $\mathbb{R}^{n}$ cut by the coordinate hyperplanes, or some arc of a (one-dimensional) circle.

Suppose that $n>1$. We choose a vector $e_{i}$ of unit length in each space $W_{i}$. In the space $V=\left\langle e_{1}, \ldots, e_{n}\right\rangle_{\mathbb{C}}$ consider the action of the $(n-1)$-dimensional Lie group $H \subset \mathbf{O}(V)$ of all complex linear diagonal operators $\operatorname{diag}\left(\lambda_{1}, \ldots, \lambda_{n}\right)$ such that $\left|\lambda_{i}\right|=1$ and $\lambda_{1}^{a_{1}} \cdots \lambda_{n}^{a_{n}}=1, a_{i} \in \mathbb{N}$. The group $H$ can be identified with the subtorus of the torus $\mathbb{T}^{n}$ defined by the single character with positive integer exponents $a_{1}, \ldots, a_{n}$.

Theorem 3.4. The quotient $V / H$ is diffeomorphic to the real vector space $\mathbb{R}^{n+1}$ and every orthogonal operator $g \in N(H)$ acts on it linearly.

Proof. The spaces $W_{i}$ are one-dimensional complex representations of the group $H$. If two of them, $W_{i}$ and $W_{j}$, are isomorphic as real representations of $H$, then the corresponding 
weights (homomorphisms $H \rightarrow \mathbb{T}$ ) coincide up to pointwise inversion. This means that the character $\left(\lambda_{1}, \ldots, \lambda_{n}\right) \rightarrow \lambda_{i} \lambda_{j}^{ \pm 1}$ of the torus $\mathbb{T}^{n}$ is trivial on the subtorus $H$; that is, its exponents have the form $c a_{1}, \ldots, c a_{n}$, where $c \in \mathbb{Z}$, so $n=2$ and $a_{1}=a_{2}=1$.

First suppose that the real representations $W_{i}$ of the group $H$ are pairwise nonisomorphic. Then they are isotypic components of the representation $H: V$ and are therefore permuted by any operator in $N(H)$.

Let us determine the structure of the quotient $V / H$. If two vectors in the space $V$ belong to the same orbit of the action $H: V$, then obviously the absolute values of their coordinates are pairwise equal and the products of their coordinates, when raised to the powers $a_{i}$ are also equal. If there are two vectors whose coordinates have pairwise equal absolute values, and zero is among these coordinates, then they belong to the same orbit. Indeed, the diagonal element corresponding to a zero coordinate can be chosen arbitrarily; therefore we may first choose them all apart from this one (there may be nonuniqueness here) and then choose the remaining one, guided by the condition $\lambda_{1}^{a_{1}} \cdots \lambda_{n}^{a_{n}}=1$. If the absolute values of the coordinates are pairwise equal and are all nonzero, then two vectors belong to the same orbit if and only if the products of coordinates raised to the powers $a_{i}$ are equal. We construct a factorization map

$$
\pi_{H}: V \rightarrow \mathbb{R}^{n+1}=\mathbb{R}^{n-1} \oplus \mathbb{C}
$$

as follows: with every vector $z=\left(z_{1}, \ldots, z_{n}\right)$ we associate the vector

$$
\left(\left|z_{1}\right|^{2}, \ldots,\left|z_{n}\right|^{2}\right) \in \mathbb{R}^{n}
$$

which is projected onto the hyperplane $\sum_{i=1}^{n} x_{i}=0$ along the vector $(1, \ldots, 1)$, that is, orthogonally. The resulting projection is by definition the coordinate of the vector $\pi_{H}(z)$ in $\mathbb{R}^{n-1}$; the coordinate in $\mathbb{C}$ is the number $z_{1}^{a_{1}} \cdots z_{n}^{a_{n}}$. The map $\pi_{H}$ thus constructed is obviously smooth and constant on orbits of $H$. On the other hand, if we know that the coordinate in $\mathbb{R}^{n-1}$ (namely, the tuple of the squares of the absolute values of the coordinates, up to the addition of the same number), as well as the product of these absolute values raised to the powers $a_{i}$, then the absolute values themselves can be uniquely reconstructed: as all the absolute values are strictly increasing, when we raise them to the powers $a_{i}$ and take their product, it is also strictly increasing, and the extreme values are 0 and $+\infty$. Hence the map $\pi_{H}$ is surjective and separates orbits.

Now consider an arbitrary element $g \in N(H)$. We already know that it permutes the subspaces $W_{i}$. It can be represented as a composite $g=g_{1} \circ g_{2} \circ g_{3}$ of $\mathbb{R}$-linear transformations: $g_{1}$ results in some permutation $\sigma$ of the complex coordinates, that is, $\left(g_{1} z\right)_{i}:=z_{\sigma(i)} ; \quad g_{2}$ takes the complex conjugates of some coordinates preserving the others; $g_{3}$ multiplies a coordinate $z_{i}$ by a number $\nu_{i},\left|\nu_{i}\right|=1$. Obviously, $g_{3} H g_{3}^{-1}=$ $H=g \mathrm{Hg}^{-1}$; therefore the operator $g_{0}:=g_{1} g_{2}$ also belongs to the normalizer of $H$. But $g_{0} \mathrm{Hg}_{0}^{-1}$ is the group of all the diagonal operators $\operatorname{diag}\left(\lambda_{1}, \ldots, \lambda_{n}\right)$ such that $\left|\lambda_{i}\right|=1$ and $\lambda_{1}^{b_{1}} \cdots \lambda_{n}^{b_{n}}=1$, where $b_{i}=a_{\sigma(i)}\left(\right.$ or $\left.b_{i}=-a_{\sigma(i)}\right)$ if the $\sigma(i)$ th coordinate is preserved (taken to the complex conjugate, respectively) by the operator $g_{2}$. Since $g_{0} H g_{0}^{-1}=H$, the numbers $b_{1}, \ldots, b_{n}$ are proportional to the numbers $a_{1}, \ldots, a_{n}$, whence

$$
a_{i}=a_{\sigma(i)} \forall i
$$

while the operator $g_{2}$ is either the identity or takes the complex conjugates of all coordinates.

Now let us see how such an element $g$ acts on the quotient. The coordinate in $\mathbb{R}^{n-1}$ changes only under the action of $g_{1}$, and in this case, the absolute values of coordinates are permuted, the linear transformation of a permutation of coordinates takes place in $\mathbb{R}^{n}$, and we simply have to restrict it to the invariant hyperplane $x_{1}+\cdots+x_{n}=0$. As for the coordinate in $\mathbb{C}$, it is multiplied by $\nu_{1}^{a_{1}} \cdots \nu_{n}^{a_{n}}$ under $g_{3}$, is preserved or goes 
to the complex conjugate under $g_{2}$, and is preserved under $g_{1}$ according to (3.3). Thus, the element $g$ acts on $V / H$ linearly.

Now suppose that among the real representations $H: W_{i}$, at least two are isomorphic. As we know, $n=2$ and $a_{1}=a_{2}=1$ in this case.

If we denote the vector $e_{1}$ by the symbol $\mathbf{1}, i e_{1}$ by the symbol $\mathbf{i}$, the vector $e_{2}$ by $\mathbf{j}$, and $i e_{2}$ by $\mathbf{k}$, then the space $V$ is identified with the space $\mathbb{H}$ of quaternions, and left multiplication by $\mathbf{i}$ here, as is easy to see, will coincide with multiplication by $i$ in the space over $\mathbb{C}$. If $v \in\langle\mathbf{j}, \mathbf{k}\rangle$, then $v \mathbf{i}=-\mathbf{i} v$, whence $v z=\bar{z} v$ for $z \in \mathbb{C}$ (complex numbers are identified with quaternions in $\langle\mathbf{1}, \mathbf{i}\rangle)$. Thus, left multiplication by a complex number $z$ is precisely multiplication by the element $z$ of the field $\mathbb{C}$ in the space over this field, and right multiplication by $z \in \mathbb{C}$ is the diagonal operator $\operatorname{diag}(z, \bar{z})$ in complex space. Consequently, the group $H$ acts by right multiplications by complex numbers with absolute value 1 . Consider the map $\pi_{H}: \mathbb{H} \rightarrow \mathbb{H}, v \rightarrow v \mathbf{i} \bar{v}$ (here conjugates are taken in the quaternion sense: the signs of all the imaginary parts are changed and the real part is preserved). It is easy to see that $\pi_{H}(\mathbb{H})=\langle\mathbf{i}, \mathbf{j}, \mathbf{k}\rangle$, while $\pi_{H}\left(v_{1}\right)=\pi_{H}\left(v_{2}\right)$ if and only if $v_{1}=v_{2} z$, where $z$ is a complex number with absolute value 1 . Thus, the fibres of the map $\pi_{H}$ are the orbits of the group $H$; therefore the quotient $V / H$ is diffeomorphic to $\mathbb{R}^{3}$.

The normalizer of the subgroup $H$ in the group of orthogonal operators is generated by $H$, with left multiplication by quaternions with norm 1 , and right multiplication by $\mathbf{j}$; on the quotient $V / H$ the normalizer acts as the group $\mathbf{O}_{3}(\mathbb{R})$.

If $n=2$, then $\operatorname{dim} H=1$, and for any orthogonal operator $g \in N(H)$, the operator $\operatorname{Ad}_{\mathfrak{h}}(g)$ on the one-dimensional algebra $\mathfrak{h}:=$ Lie $H$ is equal to $( \pm E)$.

Assertion 3.1. Suppose that $n=2$ and the group $H$ is connected. Then an orthogonal operator $g \in N(H)$ acts on $V / H \cong \mathbb{R}^{3}$ by a reflection if and only if $g^{2}=E$ and $\operatorname{Ad}_{\mathfrak{h}}(g)=-E$.

Proof. If $\operatorname{Ad}_{\mathfrak{h}}(g)=\varepsilon E, \varepsilon= \pm 1$, then the determinant of the operator $g$ on the space $V / H$ is equal to $\varepsilon$. Therefore we can assume that $\operatorname{Ad}_{\mathfrak{h}}(g)=-E$. In this case, in the space $V$, any operator in $\mathfrak{h} \backslash\{0\}$ is nonsingular and anticommutes with $g$, so

$$
\operatorname{dim}(\operatorname{Ker}(E-g))=\operatorname{dim}(\operatorname{Ker}(E+g))=\frac{1}{2} \operatorname{dim}\left(\operatorname{Ker}\left(E-g^{2}\right)\right) .
$$

Furthermore, every orbit of the group $H$ that is invariant under $g$ has a nonempty finite intersection with the subspace $\operatorname{Ker}(E-g)$. Therefore the dimensions of the fixed point subspaces for the action of $g$ on the spaces $V$ and $V / H$ coincide, that is,

$$
\operatorname{dim}(V / H)^{g}=\operatorname{dim}(\operatorname{Ker}(E-g))=\frac{1}{2} \operatorname{dim}\left(\operatorname{Ker}\left(E-g^{2}\right)\right) .
$$

In particular, $\operatorname{dim}(V / H)^{g}=2 \Leftrightarrow g^{2}=E$.

Lemma 3.2. Let $G$ be a finite subgroup of $\mathbf{O}_{3}(\mathbb{R})$ containing at least one reflection. Then for its tautological representation in $V:=\mathbb{R}^{3}$ all the homotopy groups of the quotient $(V \backslash\{0\}) / G$ are trivial.

Proof. It is sufficient to prove that all the homotopy groups of the quotient of the unit sphere $S \subset V$ by the action of $G$ are trivial.

By hypothesis, the subgroup $H \subset G$ generated by all the reflections of the group $G$ is nontrivial. Therefore there exists a fundamental domain $M \neq S$ for its action on $S$, which is a closed convex spherical polygon homeomorphic to a disc. We have $G=H \lambda \Delta$, where $\Delta:=\{g \in G: g M=M\}$. The group $\Delta$ contains no reflections and leaves the centre of the spherical polygon $M$ invariant; therefore it acts on $M$ by rotations. The 
quotient $M / \Delta$ is also homeomorphic to a disc; in particular, all its homotopy groups are trivial.

No two points in $M$ belong to the same orbit of the action $H: S$, so the quotient $S / G$ is homeomorphic to $M / \Delta$ and all its homotopy groups are trivial.

Theorem 3.5. Suppose that a one-dimensional group $G$ acts on a four-dimensional space $V$, and that the set $P$ contains no zero weights. Also suppose that there exists an involution $g \in G$ such that $\operatorname{Ad}(g)=-E$. Then all the homotopy groups of the quotient $(V \backslash\{0\}) / G$ are trivial.

Proof. Since $\operatorname{dim} G=1$, the group $\operatorname{Ad}(G)$ coincides with $\{ \pm E\}$.

The space $V$ can be decomposed into a direct sum of two irreducible subrepresentations of the group $G^{0}$, each of which has the structure of a one-dimensional complex space. Therefore the group $G^{0}$ embeds into the two-dimensional torus $\mathbb{T}^{2}=\mathbb{T} \times \mathbb{T}$ so that the image under the embedding does not contain any of the direct factors and therefore is defined by a single character with nonzero exponents (we can assume they are positive integers). Consequently, $V$ is a two-dimensional complex space on which $G^{0}$ acts by all the diagonal complex-linear operators $\operatorname{diag}\left(\lambda_{1}, \lambda_{2}\right)$ such that $\left|\lambda_{i}\right|=1$ and $\lambda_{1}^{a_{1}} \lambda_{2}^{a_{2}}=1$, $a_{i} \in \mathbb{N}$. By Theorem 3.4, the quotient $V / G^{0}$ is homeomorphic to the three-dimensional space $\mathbb{R}^{3}$, the factorization map takes the zero vector to the zero vector, and $G$ acts on $V / G^{0}$ linearly. An involution $g \in G$ such that $\operatorname{Ad}(g)=-E$ acts on $V / G^{0}$ by a reflection. We have

$$
V / G \cong\left(V / G^{0}\right) /\left(G / G^{0}\right), \quad\left|G / G^{0}\right|<\infty,
$$

and it remains to apply Lemma 3.2

\section{Systems of VeCtors in a finite-Dimensional SPACE}

We now examine in more detail the stability of finite sets of vectors in a space. Let $P$ be a finite set of vectors in some finite-dimensional vector space over a field $\mathbb{F}$. Some of these vectors may coincide; multiplicity is taken into account. We denote the number of nonzero vectors in the set $P$ by $\|P\|$.

Definition. A set of vectors is called trivial if all its vectors are zero.

For $q>0$, every $q$-stable set is $(q-1)$-stable. In turn, a $(q-1)$-stable set $P$ is $q$-stable if and only if, when any $q$ vectors are removed from it, a set with linear hull $\langle P\rangle$ remains.

As established in 11 if a set $P$ is nontrivial and $q$-stable, then $\|P\| \geq \operatorname{dim}\langle P\rangle+q$. Furthermore, the linear hull must not change if any $q$ nonzero vectors are removed from $P$. Consequently, when $\|P\|=\operatorname{dim}\langle P\rangle+q$, any $\operatorname{dim}\langle P\rangle$ nonzero vectors generate the space $\langle P\rangle$ linearly, and therefore they are linearly independent; therefore any set of at most $\operatorname{dim}\langle P\rangle$ nonzero vectors in $P$ is linearly independent. Hence the following assertion follows immediately.

Assertion 4.1. In a $q$-stable set $P$ satisfying the inequality $\|P\| \leq \operatorname{dim}\langle P\rangle+q$, any set of at most $\operatorname{dim}\langle P\rangle$ nonzero vectors is linearly independent.

If a $q$-stable set $P$ admits a proper decomposition into two components $P_{1}$ and $P_{2}$, then the sets $P_{i}$ are nontrivial and $q$-stable. We have the inequalities $\left\|P_{i}\right\| \geq \operatorname{dim}\left\langle P_{i}\right\rangle+q$; therefore for $q>0$ we have

$$
\begin{aligned}
\|P\| & =\left\|P_{1}\right\|+\left\|P_{2}\right\| \geq\left(\operatorname{dim}\left\langle P_{1}\right\rangle+q\right)+\left(\operatorname{dim}\left\langle P_{2}\right\rangle+q\right) \\
& =\operatorname{dim}\langle P\rangle+2 q>\operatorname{dim}\langle P\rangle+q .
\end{aligned}
$$

Hence one can derive the following assertion. 
Assertion 4.2. If $q>0$, then any $q$-stable set $P$ satisfying $\|P\| \leq \operatorname{dim}\langle P\rangle+q$ is indecomposable.

We will primarily be interested in 1-stable and 2-stable sets.

A set $P$ is 1-stable if and only if any vector in $P$ can be expressed linearly in terms of the others. Every 2-stable set is 1-stable, but the converse is false. We give a criterion for a 1-stable set of vectors $P$ to be 2 -stable.

Consider two arbitrary vectors in a set $P$ (it is not necessarily 1-stable). We say they are equivalent if and only if, in any linear relation between vectors in $P$, either the coefficients of both vectors or of neither vector vanish. Clearly, the resulting relation between vectors in $P$ is an equivalence relation. It is invariant under multiplication of some vectors by a nonzero scalar. Each zero vector in $P$ can be equivalent only to itself. Therefore if an equivalence class contains more than one vector, then all of them are nonzero. As is easy to see, removing and adding zero vectors does not affect equivalence in the set $P$.

Theorem 4.1. An arbitrary 1-stable set is 2-stable if and only if all of its elements are pairwise nonequivalent.

Proof. Suppose that $P$ is a 2 -stable set. We claim that no two vectors $a, b \in P$ are equivalent. Indeed, if we remove the vectors $a$ and $b$ from $P$ (counting the multiplicities of all vectors), then a set $P^{\prime}$ must be left such that $\left\langle P^{\prime}\right\rangle=\langle P\rangle$. Since $b \in\left\langle P^{\prime}\right\rangle$, there exists a linear relation between vectors in $P$, where $b$ has a nonzero coefficient and the coefficient of $a$ is zero. Hence $a$ and $b$ are nonequivalent.

Conversely, suppose that all elements of a 1 -stable set $P$ are pairwise nonequivalent. Let us see what happens when we remove two arbitrary vectors $a, b \in P$ from it. Since $a$ and $b$ are nonequivalent, there exists a linear relation between vectors in $P$ in which, without loss of generality, the coefficient of $a$ is zero, and of $b$ is nonzero (or possibly vice versa). Then when we remove $a$ and $b$ from $P$, a set $P^{\prime}$ is left, whose linear hull contains $b$. Therefore, $\left\langle P^{\prime}\right\rangle=\left\langle P^{\prime} \cup\{b\}\right\rangle=\langle P \backslash\{a\}\rangle$, and since $P$ is a 1-stable set, $\langle P \backslash\{a\}\rangle=\langle P\rangle$, whence $\left\langle P^{\prime}\right\rangle=\langle P\rangle$.

Every equivalence class $N$ of an arbitrary set $P$ consisting of more than one vector, together with any of its vectors $v$, also contains all its copies in $P$. Indeed, if $w \in P$ is another copy of the vector $v$, then in view of the linear relation $v-w=0$, none of the vectors in $P$ apart from $v$ and $w$ is equivalent to $v$, that is, $N \subset\{v ; w\}$. Since $\|N\|>1$, we have $w \in N$.

Let $P_{1}$ and $P_{2}$ be finite sets of vectors in finite-dimensional spaces over a field $\mathbb{F}$ such that $P_{2}$ is obtained from $P_{1}$ by a linear isomorphism $A$, up to nonzero scalars: $P_{2}=\left\{\varepsilon_{v} A v: v \in P_{1}\right\}, \varepsilon_{v} \in \mathbb{F}^{*}$. Then any equivalence class $N_{1}$ of the set $P_{1}$ goes under the action of $A$ to some equivalence class $N_{2}$ of the set $P_{2}$, up to nonzero scalars: $\varepsilon_{v} A v \in N_{2} \Leftrightarrow v \in N_{1}$.

Consider a set $P$ and an equivalence class $N$ in it. If $P$ is a 1-stable set, then there exists a linear relation $\sum_{v \in P} c_{v} v=0$ where some vector $w \in N$ has a nonzero coefficient $c_{w}$. Then $\sum_{v \in N} c_{v} v \in\langle P \backslash N\rangle$ is a nontrivial linear combination of vectors in $N$ that belongs to the linear hull of the others. In any such nontrivial linear combination, all the coefficients $c_{v}, v \in N$, are nonzero, since $N$ is an equivalence class. Therefore, up to proportionality, there is exactly one such a nontrivial linear combination with coefficients $c_{v} \neq 0$.

In what follows we shall be interested only in the case $\mathbb{F}=\mathbb{R}$, although up to now this has not been used anywhere. Let $N \subset P$ be an equivalence class. By changing the sign of some elements of this class, if necessary, we can ensure that a nontrivial linear combination of vectors in $N$ contained in the linear hull of the others has coefficients $c_{v}$ 
$(v \in N)$ with the same sign; we can assume they are positive. This procedure can be performed independently with each class $N$, and then for all classes we shall have $c_{v}>0$, $v \in N$. Here, the set $P$ does not have to go to itself (the signs may change). Changing signs preserves the 1-stability of a set, as well as the orders of all equivalence classes.

\section{A Representation with a 2-Stable Set of Weights}

In this section we assume that for a given action $G: V$ the set $P$ of weights corresponding to irreducible components, accounting for multiplicities, is 2-stable. As we already know, on the space $\mathfrak{g}$ there exists an inner product invariant under $\operatorname{Ad}(G)$.

If $v \in V$ is an arbitrary vector, then the set of weights corresponding to the representation $G_{v}: V$ consists precisely of the restrictions of the weights of the 2-stable set $P$ to the subspace $\mathfrak{g}_{v} \subset \mathfrak{g}$ and therefore is 2-stable. The representation $G_{v}: N_{v}$ also has a corresponding 2-stable set of weights, since the group $G_{v} \cap G^{0}$ acts trivially on $\mathfrak{g} v$.

We consider some element $g \in \Omega$ and set $A:=\operatorname{Ad}(g)$, which is a linear operator on the space $\mathfrak{g}, P^{A}:=P \cap \operatorname{Ker}(E-A)$, and $r:=\operatorname{rk}(E-A)$. The operator $g$ in the space $V$ leaves all the isotypic components of the representation $G^{0}: V$ invariant if and only if $P \subset \operatorname{Ker}(E-A) \cup \operatorname{Ker}(E+A)$, which is equivalent to

$$
P \backslash P^{A} \subset \operatorname{Ker}(E+A) .
$$

Theorem 5.1. Suppose that $A \neq E$. Then

1) the set $(E-A) P$ contains exactly $r+2$ nonzero vectors, is indecomposable, and any collection of at most $r$ nonzero vectors is linearly independent;

2) the operator $g$ acts trivially on all the isotypic components $V_{\lambda}, \lambda \in P^{A}$, including $V_{0}$;

$3)$ if the set $P \backslash P^{A}$ is not contained in the subspace $\operatorname{Ker}(E+A)$, then it consists of exactly three weights $\lambda^{+}, \lambda^{-}, \lambda$, each of which occurs in $P$ with multiplicity 1 ; furthermore, $A \lambda=-\lambda$,

$$
A \lambda^{ \pm}=\lambda^{\mp} \neq \pm \lambda^{ \pm}
$$

and the operator $A$ is the reflection with respect to the hyperplane $\langle\lambda\rangle^{\perp}$.

Proof. Since $A \neq E$, the number $r$ is positive. The set $(E-A) P$, like $P$, is 2-stable. Its linear hull $(E-A)\langle P\rangle=(E-A) \mathfrak{g}$ has dimension $r>0$, whence $\|(E-A) P\| \geq r+2$. We have

$$
\operatorname{rk}(E-g)=\operatorname{rk}(E-A)+\omega(g) \leq r+2 \leq\|(E-A) P\| .
$$

The operator $g$ in the space $V$ permutes the isotypic components of the representation $G^{0}: V$. Consider an independent cycle of this permutation: $V_{\lambda}, g V_{\lambda}=V_{A \lambda}, \ldots, g^{k} V_{\lambda}=$ $V_{A^{k} \lambda}$, where $k \geq 0, \quad k+1$ is the length of the cycle, and $g^{k+1} V_{\lambda}=V_{\lambda}$. Each $g^{i} V_{\lambda}$ is a direct sum of the same number of irreducible components, which is denoted by $l$ (for different cycles, $l$ may be different). Among the weights $\lambda, A \lambda, \ldots, A^{k} \lambda \in P$, no two are equal or opposite, each has multiplicity $l$, and $A^{k+1} \lambda= \pm \lambda$, that is, $A^{k+1} \lambda=(-1)^{\varepsilon} \lambda$, where $\varepsilon=0,1$ (if $\lambda=0$, then we set $\varepsilon:=0$ ). Let $Q \subset P$ be the subset consisting of the weights $\lambda, A \lambda, \ldots, A^{k} \lambda$, each of which is taken with multiplicity $l$. Clearly,

$$
V_{Q}=\bigoplus_{i=0}^{k} g^{i} V_{\lambda} \quad \text { and } \quad g V_{Q}=V_{Q}
$$

Suppose that $\lambda \neq 0$. Then the kernel of the restriction of the operator $E-g$ to $V_{Q}$ has dimension at most $2 l$. Indeed, if

$$
(E-g)\left(v_{0}+\cdots+v_{k}\right)=0, \quad v_{i} \in g^{i} V_{\lambda},
$$


then $g v_{i}=v_{i+1}(i<k), g v_{k}=v_{0}$, and $g^{k+1} v_{0}=v_{0}$. Consequently, if $v_{0}=0$, then all the $v_{i}$ are also equal to zero, that is, the kernel is projected onto $V_{\lambda}$ injectively. Furthermore, its dimension is equal to the dimension of the kernel of the restriction of $E-g^{k+1}$ to $V_{\lambda}$. If in addition $\varepsilon=1$, so that $A^{k+1} \lambda=-\lambda$, then the restriction of $g^{k+1}$ to $V_{\lambda}$ is antilinear; therefore the kernels of the restrictions to $V_{\lambda}$ of the operators $E-g^{k+1}$ and $E+g^{k+1}$ are obtained from each other by multiplication by $i$, have the same dimension, and have trivial intersection. Therefore, neither has dimension greater than $l$. Thus, the dimension of the kernel of the restriction of $E-g$ to the subspace $V_{Q}$ does not exceed $2 l$, and for $\varepsilon=1$ it does not exceed $l$; in either case this dimension does not exceed $2 l-\varepsilon l$. Hence,

$$
\operatorname{dim}\left((E-g) V_{Q}\right) \geq \operatorname{dim} V_{Q}-(2 l-\varepsilon l)=2 l(k+1)-(2 l-\varepsilon l)=2 k l+\varepsilon l .
$$

The set $Q$ contains $(k+1) l$ weights accounting for multiplicities; consequently,

$$
\|(E-A) Q\| \leq(k+1) l
$$

and

$$
\operatorname{dim}\left((E-g) V_{Q}\right)-\|(E-A) Q\| \geq 2 k l+\varepsilon l-(k+1) l=(k-1+\varepsilon) l .
$$

The number $k-1+\varepsilon$ can be negative only under the condition $k=\varepsilon=0$ or, equivalently, $A \lambda=\lambda$. Thus, if $A \lambda \neq \lambda$, then automatically $\lambda \neq 0$ and

$$
\operatorname{dim}\left((E-g) V_{Q}\right) \geq\|(E-A) Q\| .
$$

But if $A \lambda=\lambda$, then $Q$ only contains the weight $\lambda$ (with multiplicity $l$ ), the set $(E-A) Q$ is trivial, $\|(E-A) Q\|=0$, and inequality (5.5) again holds.

By summing the inequalities (5.5) over all independent cycles of the permutation of the set of isotypic components, we obtain the inequality $\operatorname{rk}(E-g) \geq\|(E-A) P\|$, since the space $V$ is the direct sum of the subspaces $V_{Q}$ and the set $P$ is the union (counting multiplicities) of the subsets $Q$. Consequently, all the inequalities in (5.3), as well as all inequalities (5.5) become equalities; in particular, $\|(E-A) P\|=r+2$. The linear hull of the 2-stable set $(E-A) P$ has dimension $r$; therefore, according to Assertions 4.2 and 4.1. this set is indecomposable and any collection of at most $r$ nonzero vectors in it is linearly independent.

As we already mentioned, for each independent cycle, $\operatorname{dim}\left((E-g) V_{Q}\right)=\|(E-A) Q\|$. If $\lambda \neq 0$ for some cycle, then $k+\varepsilon \leq 1$ by (5.4). This last inequality is also true for $\lambda=0$, since in this case $A \lambda=\lambda$ and $k=\varepsilon=0$. For every $\lambda \in P^{A}$ the isotypic component $V_{\lambda}$ is invariant under the action of $g$ and forms an independent cycle by itself. Furthermore, the set $(E-A) Q$ is trivial, whence $\operatorname{dim}\left((E-g) V_{Q}\right)=\|(E-A) Q\|=0$; that is, $g$ acts trivially on $V_{Q}=V_{\lambda}$.

Now suppose that the inclusion (5.1) does not hold. Then the operator $g$ yields a nonidentity permutation on the set of isotypic components. This permutation has an independent cycle such that $k>0$, and since $k+\varepsilon \leq 1$, we have $k=1$ and $\varepsilon=0$. This means that the components $V_{\lambda^{+}}$and $V_{\lambda^{-}}$occur in this cycle, while the weights $\lambda^{ \pm}$satisfy (5.2). The two nonzero vectors $(E-A) \lambda^{ \pm}=\lambda^{ \pm}-\lambda^{\mp}$ in the set $(E-A) P$ are opposite to each other and therefore linearly dependent. Consequently, $r=1$, $\|(E-A) P\|=r+2=3$, the operator $A$ is a reflection, and the set $P \backslash P^{A}$ consists of exactly three weights, counting multiplicities. The weights $\lambda^{+}$and $\lambda^{-}$belong to $P \backslash P^{A}$; therefore $P \backslash P^{A}=\left\{\lambda^{+}, \lambda^{-}, \lambda\right\}$ and the weights $\lambda^{+}, \lambda^{-}$and $\lambda$ occur in $P$ with multiplicity 1 . All the isotypic components corresponding to the weights of the set $P^{A}$ are invariant under the action of $g$, while $V_{\lambda^{+}}$ and $V_{\lambda^{-}}$change places. The remaining component $V_{\lambda}$ is invariant under the action of $g$; hence $A \lambda=-\lambda$ and $A$ is a reflection with respect to the hyperplane $\langle\lambda\rangle^{\perp}$.

Corollary 5.1. Every operator $g \in \Omega$ leaves all the isotypic components $V_{\lambda}$ invariant, possibly apart from two of them. 
Corollary 5.2. If $\operatorname{Ad}(\Omega) \ni-E$, then $\|P\|=m+2$.

Thus, if the inclusion (5.1) does not hold, then the given element $g \in \Omega$ has a corresponding triple of weights $\left\{\lambda^{+}, \lambda^{-}, \lambda\right\}$, each of which occurs in $P$ with multiplicity 1. Furthermore,

$$
(E+A)\left(\lambda^{+}-\lambda^{-}\right)=\left(\lambda^{+}-\lambda^{-}\right)+\left(\lambda^{-}-\lambda^{+}\right)=0
$$

therefore,

$$
0 \neq \lambda^{+}-\lambda^{-} \in \operatorname{Ker}(E+A)=\langle\lambda\rangle .
$$

Thus, between the weights $\lambda^{+}, \lambda^{-}$, and $\lambda$ there exists a linear relation, all three of whose coefficients are nonzero. In particular, any subspace of $\mathfrak{g}$ containing two vectors in the triple $\left\{\lambda^{+}, \lambda^{-}, \lambda\right\}$ contains the whole triple. Note that

$$
\operatorname{dim}\left\langle\lambda^{+}, \lambda^{-}, \lambda\right\rangle=2
$$

that is, the linear hull of this triple is two-dimensional: according to (5.2), the weights $\lambda^{ \pm}$do not belong to the line $\operatorname{Ker}(E+A)=\langle\lambda\rangle$.

Theorem 5.2. All the indecomposable components of the set $P$ are entirely contained in $P^{A}$, possibly apart from one of them.

Proof. If $A=E$, then there is nothing to prove.

Suppose that $A \neq E$ and that (5.1) holds. Then the sets $P^{A}$ and $P \backslash P^{A}$ are contained in the subspaces $\operatorname{Ker}(E-A)$ and $\operatorname{Ker}(E+A)$, respectively, which have trivial intersection. Therefore, each of these sets is a union of some indecomposable components of the set $P$. By Theorem [5.1, the set $(E-A) P$ is indecomposable. It coincides (up to zeros) with the set $2\left(P \backslash P^{A}\right)$; therefore the set $P \backslash P^{A}$ is also indecomposable and may include at most one indecomposable component of $P$. The other indecomposable components are completely contained in $P^{A}$. Note that if $P$ is indecomposable, then (up to zero weights) one of the sets $P^{A}$ and $P \backslash P^{A}$ is empty, and the other coincides with $P$, and since $\langle P\rangle=\mathfrak{g}$ and $A \neq E$, we have $A=-E$.

On the other hand, if the inclusion (5.1) does not hold, then the element $g \in \Omega$ has a corresponding triple of weights $\left\{\lambda^{+}, \lambda^{-}, \lambda\right\} \subset P$. These three weights belong to the same indecomposable component of the set $P$, since there exists a linear relation between them with all three coefficients nonzero. All the other indecomposable components are entirely contained in $P^{A}$.

We return to the set $L$ of subspaces of the space $V$ defined in $\$ 1$,

Lemma 5.1. Every operator $g \in \Omega$ acts trivially on all the subspaces in the set $L$, possibly apart from one of them. In particular, $g V_{\Lambda}=V_{\Lambda}$ for all indecomposable components $\Lambda \subset P$

Proof. For $\operatorname{Ad}(g) \neq E$, the assertion follows from Theorems 5.2 and 5.1 all the indecomposable components of $P$, except for one of them, are contained in $P^{A}$, and $g$ acts trivially on all the $V_{\lambda}$ for $\lambda \in P^{A}$, including $V_{0}$.

If $\operatorname{Ad}(g)=E$, then $\operatorname{rk}(E-g)=\omega(g) \leq 2$. The operator $g$ leaves all the subspaces $V_{\lambda}$ invariant, and acts on each of them, except for $V_{0}$, complex-linearly. Next, we have $V=\bigoplus_{\lambda} V_{\lambda}$ and

$$
\operatorname{rk}(E-g)=\sum_{\lambda} \operatorname{dim}\left((E-g) V_{\lambda}\right)=\sum_{\lambda \neq 0} 2 \operatorname{dim}_{\mathbb{C}}\left((E-g) V_{\lambda}\right)+\operatorname{dim}\left((E-g) V_{0}\right) .
$$

Therefore at most one of the numbers $\operatorname{dim}_{\mathbb{C}}\left((E-g) V_{\lambda}\right), \lambda \neq 0$, and $\operatorname{dim}\left((E-g) V_{0}\right)$ can be nonzero. Consequently, there exists at most one isotypic component $V_{\lambda}$ on which $g$ does not act trivially. 
We now prove Theorem 1.2 ,

Suppose that $V / G$ is a smooth manifold. The direct product of the subgroups $G[W]$, $W \in L$, contains the subgroup $G^{0}$, as well as the set $\Omega$ : by Lemma 5.1 , each element of $\Omega$ belongs to some direct factor. Therefore, according to Corollary 2.8, this direct product coincides with $G$. In particular,

$$
G=G[W] \times G\left[W^{\perp}\right]
$$

for any $W \in L$.

We fix a subspace $W \in L$. By Assertion 2.2, there exists a vector $v \in W^{\perp}$ such that $M_{v} \subset W$. Then $N_{v} / G_{v}$ is a smooth manifold (Theorem 2.1). At the same time, $\mathfrak{g} v \subset W^{\perp}, N_{v} \supset W, N_{v}=W \oplus\left(N_{v} \cap W^{\perp}\right)$, and the group $G_{v}$ acts trivially on the second direct summand. Hence for $d:=\operatorname{dim}\left(N_{v} \cap W^{\perp}\right)$, the quotient $\left(W \oplus \mathbb{R}^{d}\right) / G_{v}$ is a smooth manifold. Furthermore, the stabilizer $G_{v}$ contains the subgroup $G[W]$ and, according to (5.8), coincides with the direct product of the subgroups $G[W]$ and $G_{v} \cap G\left[W^{\perp}\right]$, and the latter acts trivially on $W$. Consequently, $\left(W \oplus \mathbb{R}^{d}\right) / G[W]$ is a smooth manifold.

Thus Theorem 1.2 is proved.

Theorem 5.3. If $\operatorname{dim} G=1$ and $\operatorname{Ad}(G)=\{E\}$, then $V / G$ is not a smooth manifold.

Proof. The set $P$ has a one-dimensional linear hull; hence it is indecomposable and nontrivial. Therefore, $\|P\| \geq \operatorname{dim}\langle P\rangle+2=3$ and the set $L$ may include only two subspaces: $V_{0}$ and $W:=V_{0}^{\perp}$. By Theorem 1.2 it is sufficient to prove that the quotient $\left(W \oplus \mathbb{R}^{d}\right) / G[W]$ is not a smooth manifold for any $d$. In turn, this follows from Theorem 3.2 the group $G[W]$ containing $G^{0}$ is one-dimensional, the action $G[W]: W$ has at least three corresponding nonzero weights and no zero weights, and $\operatorname{Ad}(G[W])=$ $\operatorname{Ad}(G)=\{E\}$.

Corollary 5.3. If $\operatorname{dim} G=1$ and $\operatorname{Ad}(G)=\{E\}$, then the quotient $\left(V \oplus \mathbb{R}^{d}\right) / G$ is not a smooth manifold for any $d$.

Proof. The representation (1.1) satisfies the hypotheses of Theorem 5.3, and the corresponding set of weights is 2 -stable.

\section{The PRoOF OF THE MAIN THEOREM}

Now we will start proving Theorem 1.3. We divide the proof into two parts. In the first part, we prove that for $\operatorname{dim} G>1$, conditions 1) 3) are necessary for the quotient $\left(V \oplus \mathbb{R}^{d}\right) / G$ to be a smooth manifold for some $d$ (the necessity of condition 4) follows from Corollary (2.6). In the second part, we prove that for $\operatorname{dim} G>0$, conditions 1) 4) are sufficient for the quotient $V / G$ to be a manifold.

6.1. Necessity. Suppose that the set $P$ is indecomposable, 2-stable, and contains no zero weights, while $m:=\operatorname{dim} G>1$.

We consider some element $g \in \Omega$ and set, as before, $A:=\operatorname{Ad}(g), r:=\operatorname{rk}(E-A)$, and $P^{A}:=P \cap \operatorname{Ker}(E-A)$. As we know, the set $(E-A) P$ is indecomposable and 2-stable, and any collection of at most $r$ nonzero vectors in it is linearly independent.

If the operator $A$ does not satisfy (5.1), then it is a reflection. In this case, $P \backslash P^{A}=$ $\left\{\lambda^{+}, \lambda^{-}, \lambda\right\}$, where $A \lambda=-\lambda$ and the vectors $\lambda^{+}$and $\lambda^{-}$satisfy relation (5.2). None of the vectors $\lambda^{+}, \lambda^{-}, \lambda$ is proportional to any other vector in $P$ and, in particular, each occurs in $P$ with multiplicity 1 . But if (5.1) holds, then $A= \pm E$, since $P$ is indecomposable. Thus, the set $\operatorname{Ad}(\Omega)$ can only contain the operators $( \pm E)$ and reflections with respect to hyperplanes in $\mathfrak{g}$. 
Lemma 6.1. If $\|P\|>m+2$, then every operator in $\operatorname{Ad}(\Omega)$ that is not the identity is a reflection with respect to a hyperplane, and different reflections have triples $\left\{\lambda^{+}, \lambda^{-}, \lambda\right\}$ corresponding to them that are disjoint even up to sign.

Proof. The first assertion follows from Corollary 5.2 . We now prove the second assertion.

Suppose that two different reflections $A_{i}=\operatorname{Ad}\left(g_{i}\right)\left(i=1,2, g_{i} \in \Omega\right)$ have corresponding triples $\left\{\lambda_{i}^{+}, \lambda_{i}^{-}, \lambda_{i}\right\}$ that intersect up to sign. We set $P^{A_{i}}:=P \cap \operatorname{Ker}\left(E-A_{i}\right)$. Then we have $P \backslash P^{A_{i}}=\left\{\lambda_{i}^{+}, \lambda_{i}^{-}, \lambda_{i}\right\}$. Furthermore, $\left\langle\lambda_{1}\right\rangle \neq\left\langle\lambda_{2}\right\rangle$, since the reflections $A_{i}$ with respect to the hyperplanes $\left\langle\lambda_{i}\right\rangle^{\perp}$ are distinct.

Any subspace of $\mathfrak{g}$ containing two weights from the triple $\left\{\lambda_{i}^{+}, \lambda_{i}^{-}, \lambda_{i}\right\}$ also contains the whole triple. Every vector in the set $P$ either belongs to the triple $\left\{\lambda_{1}^{+}, \lambda_{1}^{-}, \lambda_{1}\right\}$ or is orthogonal to the vector $\lambda_{1}$. Therefore the triple $\left\{\lambda_{2}^{+}, \lambda_{2}^{-}, \lambda_{2}\right\}$ is contained in the union of the subspaces $\left\langle\lambda_{1}^{+}, \lambda_{1}^{-}, \lambda_{1}\right\rangle$ and $\left\langle\lambda_{1}\right\rangle^{\perp}$. At least one of these subspaces contains at least two vectors in the triple $\left\{\lambda_{2}^{+}, \lambda_{2}^{-}, \lambda_{2}\right\}$ and therefore also this whole triple. But none of the vectors in the triple $\left\{\lambda_{1}^{+}, \lambda_{1}^{-}, \lambda_{1}\right\}$ is orthogonal to $\lambda_{1}$, while the triple $\left\{\lambda_{2}^{+}, \lambda_{2}^{-}, \lambda_{2}\right\}$ intersects it up to sign and therefore is not contained in $\left\langle\lambda_{1}\right\rangle^{\perp}$. Consequently,

$$
\left\{\lambda_{2}^{+}, \lambda_{2}^{-}, \lambda_{2}\right\} \subset\left\langle\lambda_{1}^{+}, \lambda_{1}^{-}, \lambda_{1}\right\rangle .
$$

According to (5.7) and (6.1), the triples $\left\{\lambda_{i}^{+}, \lambda_{i}^{-}, \lambda_{i}\right\}$ have the same two-dimensional linear hull $\alpha=\left\langle\lambda_{1}, \lambda_{2}\right\rangle$. In turn, the set $P^{A_{1}} \cap P^{A_{2}}$ obtained from $P$ by removing these two triples is contained in $\left\langle\lambda_{1}, \lambda_{2}\right\rangle^{\perp}=\alpha^{\perp}$. Being indecomposable, the set $P$ coincides with the union of the triples $\left\{\lambda_{i}^{+}, \lambda_{i}^{-}, \lambda_{i}\right\}, i=1,2, \mathfrak{g}=\langle P\rangle=\alpha, \quad m=\operatorname{dim} \alpha=2$, $\|P\|>m+2=4$, and $\left\|P^{A_{i}}\right\|=\|P\|-3>1$. Thus, the set $P^{A_{1}}$ contains more than one vector and is contained in the line $\left\langle\lambda_{1}\right\rangle^{\perp}$. However, each vector in $P$ belongs to some of the triples $\left\{\lambda_{i}^{+}, \lambda_{i}^{-}, \lambda_{i}\right\}$ and cannot be proportional to any other vector in the set $P$. We have obtained a contradiction.

Lemma 6.2. Suppose that every operator in $\operatorname{Ad}(\Omega)$ that is not the identity is a reflection with respect to a hyperplane, and suppose that triples $\left\{\lambda^{+}, \lambda^{-}, \lambda\right\}$ that are disjoint even up to sign correspond to different reflections there. Then the quotient $\left(V \oplus \mathbb{R}^{d}\right) / G$ is not a smooth manifold for any $d$.

Proof. Suppose that $\left(V \oplus \mathbb{R}^{d}\right) / G$ is a smooth manifold $(d \geq 0)$.

Let $\left\{\lambda_{1}, \ldots, \lambda_{p}\right\} \subset P$ be the subset of all the weights $\lambda \in P$ such that the reflection operator with respect to $\langle\lambda\rangle^{\perp}$ belongs to the set $\operatorname{Ad}(\Omega)$. For each $i=1, \ldots, p$ we denote by $g_{i}$ an element of $\Omega$ such that $\operatorname{Ad}\left(g_{i}\right)$ is its reflection with respect to the hyperplane $\left\langle\lambda_{i}\right\rangle^{\perp}$ (such an element does exist), and by $\left\{\lambda_{i}^{+}, \lambda_{i}^{-}, \lambda_{i}\right\}$ we denote the corresponding triple of weights. These $p$ triples are pairwise disjoint (even up to sign).

Each vector $\lambda_{i}$ is orthogonal to all the vectors in $P$, except for $\lambda_{i}^{+}, \lambda_{i}^{-}$, and $\lambda_{i}$; in particular, $\lambda_{i} \perp \lambda_{j}$ for $i \neq j$. The vectors $\lambda_{1}, \ldots, \lambda_{p}$ are pairwise orthogonal and therefore linearly independent, whence $p \leq m$.

Every nonidentity operator in $\operatorname{Ad}(\Omega)$ coincides with some $\operatorname{Ad}\left(g_{i}\right)$; therefore the whole group $\operatorname{Ad}(G)$ is generated by the reflections $\operatorname{Ad}\left(g_{i}\right)$ (Corollary 2.9). These reflections commute pairwise, since the $\lambda_{i}$ are pairwise orthogonal. Consequently, $\operatorname{Ad}(G)$ consists precisely of all operators of the form

$$
\left(\operatorname{Ad}\left(g_{1}\right)\right)^{\varepsilon_{1}} \cdots\left(\operatorname{Ad}\left(g_{p}\right)\right)^{\varepsilon_{p}}
$$

where each $\varepsilon_{i}$ is equal to 0 or 1 . If for $g \in G$ we represent $\operatorname{Ad}(g)$ in the form (6.2), then

$$
g=g_{1}^{\varepsilon_{1}} \cdots g_{p}^{\varepsilon_{p}} \cdot g_{0}
$$

where $\operatorname{Ad}\left(g_{0}\right)=E$. The element $g_{i}$ transposes $V_{\lambda_{i}^{+}}$and $V_{\lambda_{i}^{-}}$and leaves all the other isotypic components invariant. As for $g_{0}$, it leaves all the isotypic components invariant. Therefore, the element $g$ leaves all the isotypic components invariant, except for 
$V_{\lambda_{1}^{+}}, \ldots, V_{\lambda_{p}^{+}}, V_{\lambda_{1}^{-}}, \ldots, V_{\lambda_{p}^{-}}$, and leaves the components $V_{\lambda_{i}^{+}}$and $V_{\lambda_{i}^{-}}$invariant (transposes them) if the number $\varepsilon_{i}$ is equal to 0 (equal to 1 ).

Suppose that $p=m$. Then $\left\{\lambda_{1}, \ldots, \lambda_{m}\right\}$ is an orthogonal basis of $\mathfrak{g}$, and each vector $\lambda \in P$ is orthogonal to all the vectors of this basis, apart from, possibly, one of the $\lambda_{i}$ (if $\lambda \in\left\{\lambda_{i}^{+}, \lambda_{i}^{-}, \lambda_{i}\right\}$ ); hence $\lambda \in\left\langle\lambda_{i}\right\rangle$ for some $i$. Thus, the indecomposable set $P$ is contained in the union of pairwise orthogonal one-dimensional subspaces $\left\langle\lambda_{i}\right\rangle$, which is impossible, since $\operatorname{dim}\langle P\rangle=m>1$.

When $p<m$, the vectors $\lambda_{1}^{+}, \ldots, \lambda_{p}^{+}$do not generate $\mathfrak{g}$. But all the vectors in $P$, except for $\lambda_{1}^{-}, \ldots, \lambda_{p}^{-}$, generate $\mathfrak{g}$ linearly, since by (5.6) the vector $\lambda_{i}^{-}$can be expressed as a linear combination of the vectors $\lambda_{i}^{+}$and $\lambda_{i}$, which do not belong to the set $\left\{\lambda_{1}^{-}, \ldots, \lambda_{p}^{-}\right\}$. Therefore there exists a subset $Q \subset P$ containing all the $\lambda_{i}^{+}$, not containing any of the $\lambda_{i}^{-}$, which generates a hyperplane in $\mathfrak{g}$ linearly. Consider a vector $v \in V$ that is projected nontrivially onto the isotypic component $V_{\lambda}$ if and only if $\lambda \in Q$. The group $G_{v}$ is onedimensional, since Lie $G_{v}$ is the intersection of the kernels of the weights of the set $Q$, which generate a hyperplane in $\mathfrak{g}$ linearly. None of the elements $g \in G_{v}$ can transpose the isotypic components $V_{\lambda_{i}^{+}}$and $V_{\lambda_{i}^{-}}$, since the vector $v=g v$ is projected nontrivially onto exactly one of them. Consequently, when $\operatorname{Ad}(g)$ is represented in the form (6.2), we have $\varepsilon_{i}=0$ for all $i$, that is, $\operatorname{Ad}(g)=E$. We see that $\operatorname{dim} G_{v}=1$ and $\operatorname{Ad}\left(G_{v}\right)=\{E\}$; hence the representation $G_{v}: N_{v}$ is faithful and (as we already know) a 2-stable set of weights corresponds to it. Applying Corollary [5.3] we see that the quotient $\left(N_{v} \oplus \mathbb{R}^{d}\right) / G_{v}$ is not a smooth manifold. According to Corollary 2.2. $\left(V \oplus \mathbb{R}^{d}\right) / G$ is not a smooth manifold either.

Theorem 6.1. If $\left(V \oplus \mathbb{R}^{d}\right) / G$ is a smooth manifold for some $d$, then the action $G: V$ satisfies conditions 1 ) 3 ) of Theorem 1.3 .

Proof. As it is 2-stable, the set $P$ contains at least $m+2$ weights. By Lemmas 6.1 and 6.2. it contains exactly $m+2$ weights, that is, condition 1) holds. Therefore any collection of at most $m$ weights in the set $P$ is linearly independent. In particular, the multiplicity of each weight is equal to 1 , since $2 \leq m$. Thus, all the isotypic components are one-dimensional complex spaces $V_{\lambda}, \lambda \in P$, that are permuted by the group $G$.

Any element $g \in G$ such that $\operatorname{Ad}(g)= \pm E$ leaves each subspace $V_{\lambda}$ invariant. Consequently, $\operatorname{Ad}(G) \ni-E$ satisfies condition 3), and if $\operatorname{Ad}(G)=\{ \pm E\}$, then conditions 2) and 3) are satisfied. But if $\operatorname{Ad}(G)=\{E\}$, then $\operatorname{Ad}(\Omega)=\{E\}$ and, according to Lemma 6.2. $\left(V \oplus \mathbb{R}^{d}\right) / G$ is not a smooth manifold.

Now suppose that the group $\operatorname{Ad}(G)$ is not contained in $\{ \pm E\}$. Then the set $\operatorname{Ad}(\Omega)$ also is not contained in the group $\{ \pm E\}$ (Corollary 2.9) and there is some reflection in it. The three vectors $\lambda^{+}, \lambda^{-}, \lambda$ of the set $P$ corresponding to this reflection are linearly dependent, whence $m=2$ and $\|P\|=m+2=4$. Apart from these three vectors, in $P$ there is exactly one vector $\lambda^{\prime}$ orthogonal to $\lambda$. The two different lines $\left\langle\lambda^{+}\right\rangle$and $\left\langle\lambda^{-}\right\rangle$ on the two-dimensional plane $\mathfrak{g}$ are symmetric with respect to each of the lines $\langle\lambda\rangle$ and $\left\langle\lambda^{\prime}\right\rangle$. Therefore, among these four weights, only $\lambda$ and $\lambda^{\prime}$, and possibly $\lambda^{+}$and $\lambda^{-}$, are orthogonal. Therefore every reflection in the set $\operatorname{Ad}(\Omega)$ leaves the pair of lines $\left\{\langle\lambda\rangle,\left\langle\lambda^{\prime}\right\rangle\right\}$ invariant. In general, every operator in $\operatorname{Ad}(\Omega)$ leaves this pair of lines invariant, since if it is not a reflection, then it is equal to $( \pm E)$. According to Corollary 2.9. the whole group $\operatorname{Ad}(G)$ leaves this pair of orthogonal lines invariant. Hence, first, $G$ leaves the subspaces $V_{\lambda} \oplus V_{\lambda^{\prime}}$ and $V_{\lambda^{+}} \oplus V_{\lambda^{-}}$invariant and, second, any operator in the group $\operatorname{Ad}(G)$ is either a rotation through an angle that is a multiple of $\pi / 2$ or a reflection.

As we see, condition 2) holds; to verify condition 3) we need to prove that the group $\operatorname{Ad}(G)$ contains the operator $(-E)$. The subgroup of rotations in $\operatorname{Ad}(G)$ can only include rotations through angles that are multiples of $\pi / 2$, and if this subgroup does not contain 
$(-E)$, then it is trivial. In this case, $|\operatorname{Ad}(G)|=2$ and there is only one operator in the set $\operatorname{Ad}(\Omega)$ which is not the identity; it is a reflection. By Lemma 6.2, the quotient $\left(V \oplus \mathbb{R}^{d}\right) / G$ is not a smooth manifold, which contradicts the hypothesis.

Thus we have proved the necessity in Theorem 1.3

6.2. Sufficiency. Suppose that the action $G: V$ satisfies conditions 1) 3) of Theorem 1.3 .

By condition 1), the dimension $m$ of the group $G$ is positive. Next, there are no zero weights in the set $P$, and all the nonzero weights correspond exactly (counting multiplicities) to the subspaces $W_{i}$. These, in turn, have the structure of one-dimensional complex spaces where $G^{0}$ acts by multiplication by complex numbers with absolute value 1 . The complex structure (that is, multiplication by $i$ ) is extended to the space $V$ from the direct summands $W_{i}$. The set $P$ is 2-stable and $\|P\|=\operatorname{dim}\langle P\rangle+2$; therefore it is indecomposable, any set in it with at most $m$ elements is linearly independent, and any set in it with at least $m$ elements linearly generates $\mathfrak{g}$. Condition 2) means that the group $G$ permutes the $W_{i}$; furthermore, for $m=2$ it can only transpose $W_{1}$ with $W_{2}$, and $W_{3}$ with $W_{4}$, for $m=1$ it can only transpose $W_{2}$ with $W_{3}$, and for $m>2$ it leaves all the $W_{i}$ invariant.

Lemma 6.3. Let $v \in V$ be some vector. We represent it in the form

$$
v=\sum_{i=1}^{m+2} w_{i},
$$

where $w_{i} \in W_{i}$. We denote by $I$ the set of all numbers $i=1, \ldots, m+2$ such that $w_{i} \neq 0$. Then

1) the stabilizer $G_{v}$ is finite if and only if $|I| \geq m$;

2) if $|I| \leq m$, then $\operatorname{dim} G_{v}=m-|I|, \quad \mathfrak{g} v=\left\langle i w_{i}: i \in I\right\rangle_{\mathbb{R}}$, and there exists an element $g$ in the stabilizer $G_{v}$ such that $\operatorname{Ad}(g)=-E$ and $g W_{i}=W_{i}$ for all $i$.

Proof. Let $Q \subset P$ be the set of weights corresponding to the subspaces $W_{i}, i \in I$ (accounting for their multiplicities).

The stabilizer $G_{v}$ is finite if and only if $\langle Q\rangle=\mathfrak{g}$, which is equivalent to $|Q| \geq m$. Thus the first assertion is proved. We now prove the second.

If $|I| \leq m$, then $|Q| \leq m$ and therefore the weights in $Q$ are linearly independent and the homomorphism $\varphi_{Q}: G^{0} \rightarrow \mathbb{T}^{|I|}$ is surjective, which immediately implies the equalities $\operatorname{dim} G_{v}=m-|I|$ and $\mathfrak{g} v=\left\langle i w_{i}: i \in I\right\rangle_{\mathbb{R}}$. Consider an arbitrary element $g \in G$ such that $\operatorname{Ad}(g)=-E$ and $g W_{i}=W_{i}, i=1, \ldots, m+2$. Since $\varphi_{Q}$ is a surjective homomorphism, there exists an element $g_{0} \in G^{0}$ such that the restriction of $g g_{0}$ to each $W_{i}, i \in I$, is the reflection with respect to the real line $\mathbb{R} w_{i}$. We have $g g_{0} \in G_{v}, \operatorname{Ad}\left(g g_{0}\right)=-E$, and $\left(g g_{0}\right) W_{i}=W_{i}$ for $i=1, \ldots, m+2$.

Lemma 6.4. If $G W_{i}=W_{i}$ for all $i=1, \ldots, m+2$, then for any vector $v \in V$ with finite stabilizer we have $G_{v}=\left\langle G_{v} \cap \Omega\right\rangle$.

Proof. Since every element $g \in G$ leaves all the $W_{i}$ invariant, the operator $\operatorname{Ad}(g)$ takes each weight in $P$ to itself or to its opposite. Since the set $P$ is indecomposable, it follows that $\operatorname{Ad}(g)= \pm E$. According to condition 3), $\operatorname{Ad}(G)=\{ \pm E\}$. If $\operatorname{Ad}(g)=-E$ for some $g \in G$, then $\operatorname{rk}(E-\operatorname{Ad}(g))=m$. Furthermore, $g$ acts on each $W_{i}$ antilinearly (by a reflection); hence $\operatorname{rk}(E-g)=m+2$ and $\omega(g)=2$. If $\operatorname{Ad}\left(G_{v}\right)=\{ \pm E\}$, then the group $G_{v}$ is generated by all the elements $g$ such that $\operatorname{Ad}(g)=-E$ and therefore $\omega(g)=2, g \in \Omega$. 
Now suppose that $\operatorname{Ad}\left(G_{v}\right)=\{E\}$. Let $I$ be the set of all $i \in\{1, \ldots, m+2\}$ such that $v$ is projected nontrivially onto $W_{i}$. Since $\left|G_{v}\right|<\infty$ and $-E \notin \operatorname{Ad}\left(G_{v}\right)$, we have $|I|>m$ (Lemma 6.3). For every element $g \in G_{v}$ the operator $\operatorname{Ad}(g)$ is trivial; consequently, $g$ acts on each $W_{i}$ complex-linearly. In the case $i \in I$ the element $g$ acts trivially on the complex space $W_{i}$, since it preserves the nonzero projection of the vector $v$ onto it. Therefore the rank of the operator $E-g$ is double the number of $i \in\{1, \ldots, m+2\}$ such that $g$ acts nontrivially on $W_{i}$, which in turn does not exceed $m+2-|I| \leq 1$. Thus, $\operatorname{rk}(E-g) \in\{0 ; 2\}$ and $E-\operatorname{Ad}(g)=0$, whence $g \in \Omega$.

Lemma 6.5. Suppose $v^{\prime} \in V$ is a vector with infinite stabilizer and represent it in the form $v^{\prime}=\sum_{i=1}^{m+2} w_{i}$, where $w_{i} \in W_{i}$. Let $I$ be the set of all numbers $i=1, \ldots, m+2$ such that $w_{i} \neq 0$, and let $V^{\prime \prime}$ be the direct sum of all the $W_{i}$ over $i \notin I$. Then

1) the space $N_{v^{\prime}}$ is the direct sum of the $G_{v^{\prime}}$-invariant subspaces $V^{\prime \prime}$ and $\left\langle w_{i}: i \in I\right\rangle_{\mathbb{R}}$, the latter coincides with $i \mathfrak{g} v^{\prime}$, and $G_{v^{\prime}}$ acts trivially on it;

2 ) if the action $G: V$ satisfies all four conditions 1) 4 , in Theorem 1.3 , then the action $G_{v^{\prime}}: V^{\prime \prime}$ satisfies similar conditions (generally speaking, with a different value of $m$ ).

Proof. If $v^{\prime}=0$, then there is nothing to prove. In what follows we will assume that $v^{\prime} \neq 0$. We denote the direct sum of all the $W_{i}$ for $i \in I$ by $V^{\prime}$. Then $|I|>0$ and $V=V^{\prime} \oplus V^{\prime \prime}$.

Using Lemma 6.3 and the condition $\left|G_{v^{\prime}}\right|=\infty$, we obtain that $0<|I|<m, \operatorname{dim} G_{v^{\prime}}=$ $m-|I|, \mathfrak{g} v^{\prime}=\left\langle i w_{i}: i \in I\right\rangle_{\mathbb{R}}, \quad i \mathfrak{g} v^{\prime}=\left\langle w_{i}: i \in I\right\rangle_{\mathbb{R}}$, and $N_{v^{\prime}}=V^{\prime \prime} \oplus i \mathfrak{g} v^{\prime}$.

Every element $g \in G_{v^{\prime}}$ permutes the subspaces $W_{i}, i \in I$; these are the subspaces that $v^{\prime}$ is projected nontrivially onto. If they are permuted nontrivially, then $|I| \geq 2$ and $m=\operatorname{dim} G \leq 2$ by condition 2), that is, $m=\operatorname{dim} G \leq 2 \leq|I|<m$, which is impossible. Consequently, every element $g \in G_{v^{\prime}}$ leaves all the subspaces $W_{i}, i \in I$, invariant; therefore the projections of the vector $v^{\prime}$ onto them are invariant too, namely, all the vectors $w_{i}, i \in I$. Thus, $G_{v^{\prime}}$ acts trivially on the subspace $\left\langle w_{i}: i \in I\right\rangle_{\mathbb{R}}$; in addition, $G_{v^{\prime}} V^{\prime}=V^{\prime}$ and $G_{v^{\prime}} V^{\prime \prime}=V^{\prime \prime}$.

The space $V^{\prime \prime}$ is the direct sum of the $m+2-|I|=\operatorname{dim} G_{v^{\prime}}+2$ one-dimensional complex spaces $W_{i}, i \notin I$. Since the group $G_{v^{\prime}}^{0}$ acts on $V^{\prime}$ trivially, the representation $G_{v^{\prime}}: V^{\prime \prime}$ has finite ineffective kernel and a 2-stable set of weights corresponds to it, and also to the representation $G_{v^{\prime}}: V$. In the stabilizer $G_{v^{\prime}}$ there exists an element $g$ that leaves all the $W_{i}$ invariant and satisfies the equality $\operatorname{Ad}(g)=-E$ (again by Lemma 6.3).

The group $G$ satisfies at least one of the two conditions: $\operatorname{dim} G \leq 2$ and $G W_{i}=W_{i}$ for all $i$; therefore the corresponding condition is also valid for any subgroup of $G$, including $G_{v^{\prime}}$. Next, the group $G$ can permute the subspaces $W_{i}$ only within fixed disjoint pairs, and there are no more than two of these pairs; this property is also inherited by the action $G_{v^{\prime}}: V^{\prime \prime}$.

Thus we have proved that the action $G_{v^{\prime}}: V^{\prime \prime}$ satisfies conditions 1) 3), It remains to prove that condition 4 ) holds.

Let $v^{\prime \prime} \in V^{\prime \prime}$ be an arbitrary vector such that its stabilizer $G^{\prime}:=G_{v^{\prime}} \cap G_{v^{\prime \prime}}$ in the group $G_{v^{\prime}}$ is finite. We need to prove that the group $G^{\prime}$ is generated by elements acting on the quotient space $V^{\prime \prime} /\left(\mathfrak{g}_{v^{\prime}} v^{\prime \prime}\right)$ by a pseudoreflection or trivially. We set $v:=v^{\prime}+v^{\prime \prime}$.

Obviously, $G^{\prime} \subset G_{v}$. Furthermore, $\left|G_{v}\right|<\infty$, since the group $G_{v}^{0}$, which leaves the subspaces $V^{\prime}$ and $V^{\prime \prime}$ invariant, is contained in the finite group $G^{\prime}$. Let $\Omega_{v}:=G_{v} \cap \Omega$ be the set of all elements of the group $G_{v}$ acting on the quotient space $V /(\mathfrak{g} v)$ by a pseudoreflection or trivially. By hypothesis, $\left\langle\Omega_{v}\right\rangle=G_{v}$. 
Since the group $G^{\prime}$ is contained in $G_{v^{\prime}}$ and $G_{v}$, it acts on $i \mathfrak{g} v^{\prime}$ trivially and leaves the subspaces $V^{\prime}, V^{\prime \prime}, \mathfrak{g} v^{\prime}, \mathfrak{g} v$ and the subspace $\mathfrak{g}_{v^{\prime}} v^{\prime \prime}$ invariant. We claim that $\mathfrak{g}_{v^{\prime}} v^{\prime \prime} \subset V^{\prime \prime}$, $\mathfrak{g} v \subset \mathfrak{g} v^{\prime}+V^{\prime \prime}$, and the quotient representations $V^{\prime \prime} /\left(\mathfrak{g}_{v} v^{\prime \prime}\right)$ and $\left(\mathfrak{g} v^{\prime}+V^{\prime \prime}\right) /(\mathfrak{g} v)$ of the group $G^{\prime}$ are isomorphic. Indeed, we have $\mathfrak{g} V^{\prime} \subset V^{\prime}$ and $\mathfrak{g} V^{\prime \prime} \subset V^{\prime \prime}$, whence

$$
\begin{aligned}
& V^{\prime \prime} \supset \mathfrak{g} v \cap V^{\prime \prime}=\mathfrak{g}\left(v^{\prime}+v^{\prime \prime}\right) \cap V^{\prime \prime}=\mathfrak{g}_{v^{\prime}}\left(v^{\prime}+v^{\prime \prime}\right)=\mathfrak{g}_{v^{\prime}} v^{\prime \prime}, \\
& \mathfrak{g} v \subset \mathfrak{g} v+V^{\prime \prime}=\mathfrak{g}\left(v^{\prime}+v^{\prime \prime}\right)+V^{\prime \prime}=\mathfrak{g} v^{\prime}+V^{\prime \prime}=\mathfrak{g} v^{\prime} \oplus V^{\prime \prime},
\end{aligned}
$$

and the quotient representations $V^{\prime \prime} /\left(\mathfrak{g} v \cap V^{\prime \prime}\right)$ and $\left(\mathfrak{g} v+V^{\prime \prime}\right) /(\mathfrak{g} v)$ of the group $G^{\prime}$ are isomorphic. The group $G^{\prime}$ acts trivially on the subspace $i \mathfrak{g} v^{\prime}=\left(\mathfrak{g} v^{\prime} \oplus V^{\prime \prime}\right)^{\perp}$. Therefore $G^{\prime} \cap \Omega_{v}$ is just the set of all elements of $G^{\prime}$ that act on $\left(\mathfrak{g} v^{\prime} \oplus V^{\prime \prime}\right) / \mathfrak{g} v$, or equivalently on $V^{\prime \prime} /\left(\mathfrak{g}_{v^{\prime}} v^{\prime \prime}\right)$, by a pseudoreflection or trivially. Thus, it is required to prove that the set $G^{\prime} \cap \Omega_{v}$ generates the group $G^{\prime}$.

The group $G_{v^{\prime}}$ acts trivially on the subspace $\left\langle w_{i}: i \in I\right\rangle_{\mathbb{R}}$ and leaves all the $W_{i}, i \in I$, invariant, and it can permute only those $W_{i}$ for $i \notin I$. Therefore, $G_{v^{\prime}}$ can permute the subspaces $W_{i}$ only within a fixed pair: if there were two of these pairs, then we would have $m+2-|I| \geq 4, m+2>4, m>2$, but for $m>2$ the group $G$ does not permute the $W_{i}$ at all. Since $m+2-|I|>2$, we can assume that this pair includes the subspaces $W_{m+1}$ and $W_{m+2}$ with $m+1, m+2 \notin I$. Let $\rho$ be the homomorphism from $G$ into the cyclic group $\mathbb{Z}_{2}$ whose kernel consists precisely of all the elements which leave $W_{m+1}$ and $W_{m+2}$ invariant. Then $G_{v^{\prime}} \cap \operatorname{Ker} \rho$ is a subgroup of finite index in $G_{v^{\prime}}$, it leaves all the $W_{i}, i=1, \ldots, m+2$, invariant and its action on $V^{\prime \prime}$, like the action $G_{v^{\prime}}: V^{\prime \prime}$, satisfies conditions 1) 3) We apply Lemma 6.4 to the action of $G_{v^{\prime}} \cap \operatorname{Ker} \rho$ on $V^{\prime \prime}$ and obtain that the finite group $G^{\prime} \cap \operatorname{Ker} \rho=\left(G_{v^{\prime}} \cap \operatorname{Ker} \rho\right) \cap G_{v^{\prime \prime}}$ is generated by elements acting on $V^{\prime \prime} /\left(\mathfrak{g}_{v^{\prime}} v^{\prime \prime}\right)$ by a pseudoreflection or trivially. Therefore,

$$
\left\langle G^{\prime} \cap \Omega_{v}\right\rangle \supset\left(G^{\prime} \cap \operatorname{Ker} \rho\right) .
$$

None of the elements of the set $\Omega_{v}$ can permute the subspaces $W_{i}$ simultaneously in two pairs. Indeed, if an element $g \in \Omega_{v}$ permutes them simultaneously in two (disjoint) pairs, then $m+2 \geq 4, m \geq 2$, and among the weights of the set $P$ any two are linearly independent; in particular, the multiplicity of each of them is equal to 1 , and the $W_{i}$ are isotypic components of the representation $G^{0}: V$. According to Corollary 5.1 the operator $g \in \Omega_{v} \subset \Omega$ leaves all the isotypic components $W_{i}$, possibly apart from two, invariant.

An arbitrary element $g \in \Omega_{v}$ which is not contained in $\operatorname{Ker} \rho$ belongs to $G^{\prime}$. Indeed, it transposes the subspaces $W_{m+1}$ and $W_{m+2}$ and therefore leaves invariant all the other $W_{i}$. In particular, $g W_{i}=W_{i}$ for all $i \in I$, since $m+1, m+2 \notin I$. Hence an element $g \in G_{v}$ leaves $V^{\prime}$ and $V^{\prime \prime}$ invariant, and therefore also the vectors $v^{\prime}$ and $v^{\prime \prime}$.

Thus, $\Omega_{v} \subset\left(G^{\prime} \cup \operatorname{Ker} \rho\right)$; therefore the set $\rho\left(G^{\prime} \cap \Omega_{v}\right)$ coincides with $\rho\left(\Omega_{v}\right)$ up to the trivial element, and since $\left\langle\Omega_{v}\right\rangle=G_{v}$, we have

$$
\rho\left(\left\langle G^{\prime} \cap \Omega_{v}\right)\right\rangle=\left\langle\rho\left(G^{\prime} \cap \Omega_{v}\right)\right\rangle=\left\langle\rho\left(\Omega_{v}\right)\right\rangle=\rho\left(\left\langle\Omega_{v}\right)\right\rangle=\rho\left(G_{v}\right) \supset \rho\left(G^{\prime}\right) .
$$

This fact, as well as (6.4), implies the required equality $\left\langle G^{\prime} \cap \Omega_{v}\right\rangle=G^{\prime}$.

Lemma 6.6. The space $V$ decomposes into a direct sum of $G$-invariant subspaces $V^{\prime}$ and $V^{\prime \prime}$ such that the quotient of the unit sphere in $V^{\prime \prime}$ by the action of $G$ is homeomorphic to a closed ball and all the homotopy groups of the quotient $\left(V^{\prime} \backslash\{0\}\right) / G$ are trivial.

Proof. Let $V^{\prime}:=W_{m+1} \oplus W_{m+2}$ and $V^{\prime \prime}:=W_{1} \oplus \cdots \oplus W_{m}$; then $V=V^{\prime} \oplus V^{\prime \prime}$, where $G V^{\prime}=V^{\prime}$ and $G V^{\prime \prime}=V^{\prime \prime}$. Corresponding to the subspace $V^{\prime \prime}$ there are $m$ linearly independent weights in $P$; furthermore, $G$ permutes the subspaces $W_{1}, \ldots, W_{m}$, and for $m>2$ leaves each of them invariant. According to Theorem 3.3. the quotient of the unit sphere in $V^{\prime \prime}$ by the action of $G$ is homeomorphic to a closed $(m-1)$-dimensional ball. 
It remains to prove that all the homotopy groups of the quotient $\left(V^{\prime} \backslash\{0\}\right) / G$ are trivial. For $m \geq 2$ this follows from Theorem 3.3. the two weights in $P$ corresponding to $V^{\prime}$ are linearly independent and $G$ transposes $W_{m+1}$ and $W_{m+2}$. When $m=1$, this follows from Theorem 3.5. Indeed, two nonzero weights correspond to the action of the one-dimensional group $G$ on the four-dimensional space $V^{\prime}$. Furthermore, there exists an element $g \in G$ leaving all the $W_{i}$ invariant and such that $\operatorname{Ad}(g)=-E$. It acts on each one-dimensional complex space $W_{i}$ antilinearly, by a reflection, and is therefore involutive.

Lemma 6.7. If the action $G: V$ satisfies condition 4$)$ of Theorem 1.3 , then $V / G$ is a manifold.

Proof. We prove the assertion by induction on the (positive) dimension $m$ of the group $G$. Suppose that $m=\operatorname{dim} G>0$ and that the assertion is already proved for all groups of positive dimension less than $m$.

It is sufficient to prove that the quotient of the unit sphere $S \subset V$ by the action of $G$ is a manifold; we can then use Lemmas 2.4 and 6.6. For every vector $v \in S$ with finite stabilizer we have the equality $G_{v}=\left\langle G_{v} \cap \Omega\right\rangle$, and by Corollary 2.6, the quotient $S / G$ is a manifold locally at the point $\pi(v)$. Let us show that for any vector $v \in S$ with infinite stabilizer, the quotient of the space $N_{v}(S)=N_{v} \cap\left(T_{v} S\right)=N_{v} \cap\langle v\rangle^{\perp}$ by the action of $G_{v}$ is a manifold. By the slice theorem, this will imply that the quotient $S / G$ is a manifold locally at each point.

Suppose that $v \in S$ and $\left|G_{v}\right|=\infty$ and let $I$ be the set of numbers $i \in\{1, \ldots, m+2\}$ such that the projection of $v$ onto $W_{i}$ is nonzero, and let $V^{\prime \prime}$ be the direct sum of all the $W_{i}$ for $i \notin I$. Then $0<|I|<m$. It follows from Lemma 6.5 that $N_{v}=V^{\prime \prime} \oplus i \mathfrak{g} v$, $v \in i \mathfrak{g} v \subset N_{v}^{G_{v}}$, and the action $G_{v}: V^{\prime \prime}$ satisfies conditions 1) 4). Note that the group $G_{v}$ has positive dimension $\operatorname{dim} G_{v}=m-|I|<m$; therefore $V^{\prime \prime} / G_{v}$ is a manifold by the induction hypothesis. Furthermore, $N_{v}(S) \supset V^{\prime \prime}, N_{v}(S)=V^{\prime \prime} \oplus\left(i \mathfrak{g} v \cap\left(T_{v} S\right)\right)$, and $G_{v}$ acts trivially on the second direct summand. Therefore the quotient of $N_{v}(S)$ by the action of $G_{v}$ is a manifold, like the quotient $V^{\prime \prime} / G_{v}$.

This completes the proof of Theorem 1.3. In addition, from Lemmas 6.4 and 6.7 it is easy to derive the assertion of Theorem 1.4 .

\section{The CASE of a OnE-Dimensional Group}

This section is devoted to proving Theorems 1.5 1.8. Suppose that $m=\operatorname{dim} G=1$ and that the set $P$ contains at least 3 nonzero weights and no zero weights.

The space $V$ has a complex structure, the stabilizer of any of its nonzero vectors is finite, and every $G^{0}$-invariant real subspace of $V$ is complex. We have $\operatorname{Ad}(G) \subset\{ \pm E\}$, and an element $g \in G$ such that the operator $\operatorname{Ad}(g)$ is equal to $E(-E)$ acts linearly (antilinearly) on $V$ over the field $\mathbb{C}$. If $\left(V \oplus \mathbb{R}^{d}\right) / G$ is a smooth manifold for some $d$, then $\operatorname{Ad}(G) \neq\{E\}$ (by Corollary [5.3), that is, $\operatorname{Ad}(G)=\{ \pm E\}$. According to Corollary 2.9, $\operatorname{Ad}(\Omega) \ni-E$; therefore by Corollary 5.2, $\|P\|=3$ and $\operatorname{dim}_{\mathbb{C}} V=3$. In what follows we assume that $\operatorname{dim}_{\mathbb{C}} V=3$ and $\operatorname{Ad}(\Omega) \ni-E$; in particular, $\operatorname{Ad}(G)=\{ \pm E\}$ and $G \neq G^{0}$.

Let $G^{\prime}:=\operatorname{Ker}$ Ad $\subset G$ be the subgroup of all complex linear transformations of the group $G$. If the representation $G: V$ is irreducible, then its restriction to the subgroup $G^{\prime}$ of index 2 is either irreducible or is a direct sum of two irreducible complex subrepresentations of equal dimension. However, the second case is impossible, since the number $\operatorname{dim}_{\mathbb{C}} V=3$ is odd. Consequently, if the representation $G: V$ is irreducible, then so is $G^{\prime}: V$. The group $G$ leaves all the isotypic components of the representation $G^{0}: V$ invariant. In particular, if the representation $G: V$ is irreducible, then $V$ is an isotypic component and $G^{0}$ acts on it by scalar operators $\lambda E, \lambda \in \mathbb{T}$. 
An element $g \in G^{\prime}$ belongs to $\Omega$ if and only if $\operatorname{rk}(E-g) \in\{0 ; 2\}$, which is equivalent to $g$ being either a complex reflection or the identity operator. In turn, an element $g \in G \backslash G^{\prime}$ belongs to $\Omega$ if and only if $\operatorname{rk}(E-g) \in\{1 ; 3\}$. The subspaces $\operatorname{Ker}(E \pm g)$ have trivial intersection and are obtained from each other by multiplying by $i$; hence,

$$
g \in \Omega \Leftrightarrow \operatorname{dim}(\operatorname{Ker}(E-g))=3 \Leftrightarrow \operatorname{Ker}(E-g) \oplus \operatorname{Ker}(E+g)=V \Leftrightarrow g^{2}=E .
$$

Therefore, $\Omega \backslash G^{\prime}$ is the set of all antilinear involutions of the group $G$. This set is nonempty, since $\operatorname{Ad}(\Omega) \ni-E$, and is a union of some cosets of $G^{0}$ in $G$ : if $\operatorname{Ad}(g)=-E$, $g^{2}=E$, and $g_{0} \in G^{0}$, then $\operatorname{Ad}\left(g g_{0}\right)=-E, \quad g g_{0} g=g g_{0} g^{-1}=g_{0}^{-1}$, and $\left(g g_{0}\right)^{2}=$ $\left(g g_{0} g\right) g_{0}=E$. Consequently, $\langle\Omega\rangle \supset G^{0}$. If in addition the quotient $\left(V \oplus \mathbb{R}^{d}\right) / G$ is a smooth manifold for some $d$, then $G=\left\langle G^{0} \cup \Omega\right\rangle=\langle\Omega\rangle$ by Corollary 2.8. This proves the first assertion of Theorem 1.6.

Applying Proposition 3.1 to the group $G^{\prime}$ we obtain that all its complex reflections generate a finite subgroup $H$ that is normal in $G$. By Theorem 3.1, the quotient $V / H$ is diffeomorphic to $V$ and any element of $G^{\prime}$ (of $G \backslash G^{\prime}$ ) acts on this quotient linearly (antilinearly) over the field $\mathbb{C}$. The representation of the one-dimensional group $G / H$ in the three-dimensional complex space $V / H$ is faithful, and no zero weight corresponds to it. Furthermore, $\operatorname{Ad}(G / H)=\{ \pm E\}$. Next, the factorization map $V \rightarrow V / H$ can be given by the formula $v \rightarrow\left(f_{1}(v), f_{2}(v), f_{3}(v)\right)$, where the $f_{i}$ are homogeneous polynomials that freely generate the algebra of invariants $\mathbb{C}[V]^{H}$. If $\operatorname{deg} f_{i} \neq \operatorname{deg} f_{j}$ for $i \neq j$, then the group $G$ in its action on $V / H \cong V$ leaves all the coordinate lines invariant and, by Theorem 1.4. $V / G$ is a manifold.

Now we start the proof of Theorem 1.5.

Lemma 7.1. Suppose that the group $G$ is reducible over the field $\mathbb{R}$, is generated by the set $\Omega$, and contains no complex reflections. Then $V / G$ is a manifold.

Proof. The nonempty set $\Omega \backslash\{E\}$ of all antilinear involutions of the group $G$ is the union of some cosets of $G^{0}$ in $G$. Let $\Omega / G^{0} \subset G / G^{0}$ be the image of this set under the factorization $G \rightarrow G / G^{0}$. If the image of some subgroup of $G$ under the factorization by $G^{0}$ is generated by its intersection with $\Omega / G^{0}$, then the subgroup itself is either generated by its intersection with $\Omega$, or is nontrivial and is contained in $G^{0}$.

Since the group $G$ is reducible, $V$ is a direct sum of two $G$-invariant complex subspaces $V^{\prime}$ and $V^{\prime \prime}$ of dimensions 2 and 1 , respectively. If the two weights in $\mathfrak{g}$ corresponding to the subspace $V^{\prime}$ are neither equal nor opposite, then $V$ is the direct sum of one-dimensional $G$-invariant complex subspaces $V_{1}^{\prime}, V_{2}^{\prime}$, and $V^{\prime \prime}$, where $V_{1}^{\prime} \oplus V_{2}^{\prime}=V^{\prime}$, and the assertion follows from Theorem 1.4 Suppose that a weight $\lambda: G^{0} \rightarrow \mathbb{T}$ with multiplicity 2 corresponds to the subspace $V^{\prime}$. If $g \in G^{0}$ and $g \in \operatorname{Ker} \lambda$, then the operator $g$ is linear over $\mathbb{C}$ and acts on $V^{\prime}$ trivially, while $G$ contains no complex reflections. Hence, $\operatorname{Ker} \lambda=\{E\}$ and the stabilizer of any nonzero vector in $V^{\prime}$ intersects $G^{0}$ trivially. The set of all antilinear operators in $G$ acting involutively on $V^{\prime}$ coincides with $\Omega \backslash\{E\}$, since every antilinear orthogonal operator on the one-dimensional complex space $V^{\prime \prime}$ is involutive.

We claim that the stabilizer of any nonzero vector $v \in V$ is generated by its intersection with $\Omega$.

Suppose that $v \in V^{\prime}$. Let $\pi_{0}: V^{\prime} \rightarrow V^{\prime} / G^{0}$ be the factorization map. The quotient $V^{\prime} / G^{0}$ is homeomorphic to $\mathbb{R}^{3}$, the finite group $G / G^{0}$ acts on this quotient linearly, and the elements of the set $\Omega / G^{0}$, and only they, act on it by reflections. The quotient group $G / G^{0}$ generated by the set $\Omega / G^{0}$ acts on $V^{\prime} / G^{0}$ as a finite group generated by reflections. Therefore the stabilizer of any vector in $V^{\prime} / G^{0} \cong \mathbb{R}^{3}$ in the group $G / G^{0}$ is generated by its elements acting on $V^{\prime} / G^{0}$ by reflections, which is the same as belonging to $\Omega / G^{0}$. The image of the group $G_{v}$ under the factorization of $G$ by $G^{0}$ coincides with the stabilizer of the element $\pi_{0}(v) \in V^{\prime} / G^{0}$ in the group $G / G^{0}$ and therefore is generated 
by its intersection with $\Omega / G^{0}$. Since the group $G_{v}$ intersects $G^{0}$ trivially, it is generated by the set $G_{v} \cap \Omega$.

For a nonzero vector $v \in V^{\prime \prime}$ we have $G v=G^{0} v$ and $G=G^{0} G_{v}$; that is, the image of the subgroup $G_{v} \subset G$ under the factorization by $G^{0}$ coincides with $G / G^{0}$ and is generated by its intersection with $\Omega / G^{0}$. Consequently, $G_{v}=\left\langle G_{v} \cap \Omega\right\rangle$; for otherwise the group $G_{v}$ would be contained in $G^{0}$, which is impossible, since $G^{0} G_{v}=G \neq G^{0}$.

Now suppose that a vector $v \in V$ does not belong to any of the subspaces $V^{\prime}$ and $V^{\prime \prime}$. Then $v=v^{\prime}+v^{\prime \prime}$, where $v^{\prime} \in V^{\prime}$ and $v^{\prime \prime} \in V^{\prime \prime}$ with $v^{\prime}, v^{\prime \prime} \neq 0$. Every complex linear operator $g \in G_{v}$ leaves invariant the vectors $v^{\prime}$ and $v^{\prime \prime}$, which are linearly independent over $\mathbb{C}$. Hence the subspace $\operatorname{Ker}(E-g)$ has complex dimension at least 2 and as there are no reflections in $G$, therefore $g=E$. Thus, $G_{v} \cap G^{\prime}=\{E\}$ and $\left|G_{v}\right| \leq 2$; therefore in the group $G_{v}$ any nonidentity operator is antilinear and involutive, that is, belongs to $\Omega \backslash\{E\}$.

According to Corollary 2.6. the quotient of the unit sphere $S \subset V$ by the action of $G$ is a manifold: we have established that the stabilizer of an arbitrary vector $v \in S$ is finite and is generated by its intersection with $\Omega$. As we already mentioned, the group $G / G^{0}$ acts on the linear space $V^{\prime} / G^{0}$ as a finite group generated by reflections. In this case, the quotient $V^{\prime} / G \cong\left(V^{\prime} / G^{0}\right) /\left(G / G^{0}\right)$ is homeomorphic to a closed unbounded convex polyhedron, and the image of zero under the factorization is a boundary point. In particular, all the homotopy groups of the quotient $\left(V^{\prime} \backslash\{0\}\right) / G$ are trivial. The unit sphere in the space $V^{\prime \prime}$ is exactly one orbit of the group $G$ and its quotient by the action of $G$ consists of a single point. By Lemma 2.4, $V / G$ is a manifold.

Corollary 7.1. If the representation $(G / H):(V / H)$ is reducible and $G=\langle\Omega\rangle$, then $V / G$ is a manifold.

Proof. Since $V / G \cong(V / H) /(G / H)$, it is sufficient to prove that the action $(G / H)$ : $(V / H)$ satisfies the hypotheses of Lemma 7.1. Indeed, this representation is reducible and none of the elements of the group $G / H$ acts on $V / H$ by a complex reflection. Finally, the condition $G=\langle\Omega\rangle$ is also inherited by the group $G / H$. Indeed, under the factorization homomorphism $G \rightarrow G / H$, the image of the subset $\Omega \subset G$ is contained in the analogous subset of the group $G / H$ : antilinear involutions again go to antilinear involutions, while all the other operators in $\Omega$ belong to $H$ and go to the identity operator.

Thus, the second assertion of Theorem [1.5] is proved. We now prove the first.

Suppose that the group $G^{0}$ acts on $V$ by scalar operators. Then it is natural to consider the subgroup $K:=G^{\prime} \cap \mathbf{S L}_{\mathbb{C}}(V)$ in the group $G^{\prime}$, consisting of all its operators with complex determinant 1 . It is easy to see that $K \triangleleft G,|K|<\infty$, and $G^{\prime}=G^{0} K$. Furthermore, the absence of complex reflections in $G$ is equivalent to the fact that in the group $K$ any operator over the field $\mathbb{C}$ is either a scalar operator or has a simple spectrum. If $K$ is a group of even order, then it contains a (nonidentity) operator of order 2 , all its eigenvalues are equal to $( \pm 1)$, and their product is 1 . Such an operator is not scalar and does not have a simple spectrum.

Consequently, if $G$ contains no complex reflections and $G^{0}$ acts on $V$ by scalar operators, then the group $K$ has finite odd order and $G^{\prime}=G^{0} K$. Therefore the group $G^{\prime}$ affects only even permutations on any system of imprimitivity of the representation $G: V$. If in addition the group $G^{\prime}$ is irreducible over $\mathbb{C}$, then the group $K$, which intersects all the cosets of $G^{0}$ in $G^{\prime}$, is also irreducible. All the finite irreducible subgroups of $\mathbf{S L}_{3}(\mathbb{C})$ are described in [5, Ch. V]. In particular, all primitive groups have even order. Therefore, the group $K$ is imprimitive. It affects various even permutations on its system of imprimitivity consisting of three lines. If such a system of imprimitivity is not unique, then $K=G^{\prime}(3,3,3)$. 
Lemma 7.2. If the group $G$ is irreducible over the field $\mathbb{R}$ and contains no complex reflections, then the quotient $\left(V \oplus \mathbb{R}^{d}\right) / G$ is not a smooth manifold for any $d$.

Proof. Since $G$ is irreducible, it follows that the group $G^{\prime}$ is also irreducible, while $G^{0}$ acts on $V$ by scalar operators. Therefore, $|K|<\infty$ and $G^{\prime}=G^{0} K$. As there are no complex reflections in $G$, the group $K$ has odd order, is irreducible and imprimitive, and any of its nonscalar operators has a simple spectrum. We can assume that $K$ has the triple of coordinate lines as a system of imprimitivity, affects various even permutations on it, and contains the operator giving a cyclic permutation of the coordinates. In the group $K$, all the diagonal operators form a subgroup of index 3 , which together with any operator $\operatorname{diag}\left(\lambda_{1}, \lambda_{2}, \lambda_{3}\right)$ contains $\operatorname{diag}\left(\lambda_{2}, \lambda_{3}, \lambda_{1}\right)$ and $\operatorname{diag}\left(\lambda_{3}, \lambda_{1}, \lambda_{2}\right)$. All the operators of the group $G$ permute systems of imprimitivity of its normal subgroup $K$.

Suppose that $\left(V \oplus \mathbb{R}^{d}\right) / G$ is a smooth manifold for some $d$. We claim that $K=$ $G^{\prime}(3,3,3)$. Indeed, if the group $K$ does not coincide with $G^{\prime}(3,3,3)$, then it has exactly one system of imprimitivity consisting of three lines. Then all the operators in $G$ permute the coordinate lines $\mathbb{C} e_{i}, 1 \leq i \leq 3$. According to Corollary 2.8, there exists an operator $g \in \Omega \backslash\{E\}$ that permutes these lines nontrivially. Being an antilinear involution, it affects a transposition on the set of these lines and, without loss of generality, transposes the lines $\mathbb{C}_{2}$ and $\mathbb{C} e_{3}$. The group $K$ together with any operator $g_{1}=\operatorname{diag}\left(\lambda_{1}, \lambda_{2}, \lambda_{3}\right)$ also contains $g_{2}:=g g_{1} g^{-1}=\operatorname{diag}\left(\overline{\lambda_{1}}, \overline{\lambda_{3}}, \overline{\lambda_{2}}\right)$, as well as $g_{1} g_{2}^{-1}=\operatorname{diag}\left(\lambda_{1}^{2}, \lambda_{2} \lambda_{3}, \lambda_{2} \lambda_{3}\right)$. The operator $g_{1} g_{2}^{-1}$ has no simple spectrum and therefore is scalar: $\lambda_{1}^{2}=\lambda_{2} \lambda_{3}$ and $\lambda_{1}^{3}=\lambda_{1} \lambda_{2} \lambda_{3}=\operatorname{det} g_{1}=1$. But the operators $\operatorname{diag}\left(\lambda_{2}, \lambda_{3}, \lambda_{1}\right)$ and $\operatorname{diag}\left(\lambda_{3}, \lambda_{1}, \lambda_{2}\right)$ also belong to $K$; consequently, $\lambda_{i}^{3}=1$ for $1 \leq i \leq 3$, that is, $g_{1}^{3}=E$. Thus, the cube of any diagonal operator in $K$ gives the identity operator and has determinant 1 . Therefore, $K=G^{\prime}(3,3,3)$.

The group $K=G^{\prime}(3,3,3)$ has order 27 . Its derived subgroup coincides with the centre $\mathcal{Z}(K)$, consists of exactly three scalar operators with determinant 1 , and is isomorphic to $\mathbb{Z}_{3} \cong \mathbb{F}_{3}^{+}$, the additive group of the field $\mathbb{F}_{3}$. The quotient group $K / \mathcal{Z}(K)$ is commutative and is isomorphic to $\mathbb{Z}_{3} \times \mathbb{Z}_{3}$, the additive group of the two-dimensional space $\mathbb{F}_{3}^{2}$. Next, a nondegenerate skew-symmetric bilinear form $\wedge: F_{3}^{2} \times \mathbb{F}_{3}^{2} \rightarrow \mathbb{F}_{3}$ corresponds to the map $K \times K \rightarrow \mathcal{Z}(K)$ given by $(a, b) \rightarrow a b a^{-1} b^{-1}$. Any automorphism of the group $K$ acts on its derived subgroup $\mathcal{Z}(K) \cong \mathbb{F}_{3}^{+}$by multiplication by a number $\varepsilon \in \mathbb{F}_{3}^{*}$, and on the quotient group $K / \mathcal{Z}(K)$, as on the space $\mathbb{F}_{3}^{2}$, by a nonsingular linear transformation that multiplies the form $\wedge$ by the same number $\varepsilon \in \mathbb{F}_{3}^{*}$.

The systems of imprimitivity of the representation $K: V$ are precisely the four pairwise disjoint triples of lines. These four systems are in bijection with the four lines of the space $\mathbb{F}_{3}^{2}$. Namely, in the group $K$ the subgroup of all operators that leave invariant each line of some (fixed) system of imprimitivity has index 3, contains the centre $\mathcal{Z}(K)$, and under the factorization by the centre gives a line in $\mathbb{F}_{3}^{2}$. If $g \in G$ and $\operatorname{Ad}(g)=\varepsilon E, \varepsilon= \pm 1$, then conjugation by $g$ leaves the group $K$ invariant. The resulting automorphism of the group $K$ acts on its centre, which is isomorphic to $\mathbb{F}_{3}^{+}$, by multiplication by the number $\varepsilon \in \mathbb{F}_{3}^{*}$, while the corresponding linear transformation in $\mathbb{F}_{3}^{2}$ multiplies the form $\wedge$ by $\varepsilon \in \mathbb{F}_{3}^{*}$ and affects a permutation with sign $\varepsilon$ on the four-element set of lines in $\mathbb{F}_{3}^{2}$. The same permutation with sign $\varepsilon$ is affected by the operator $g$ on the four-element set of systems of imprimitivity of the representation $K: V$.

We fix one of the systems of imprimitivity and choose a line $\mathbb{C} v, v \neq 0$, in it. The stabilizer of the vector $v$ in the group $K$ consists of three elements. Hence the finite group $G_{v}$ is nontrivial and, by Corollary [2.6, the set $G_{v} \cap \Omega$ contains a nonidentity operator $g \in \Omega \backslash\{E\}$. We have $g(\mathbb{C} v)=\mathbb{C} v$; therefore this system of imprimitivity is preserved by the operator $g \in G \backslash G^{\prime}$, by the subgroups $K$ and $G^{0}$, and therefore also by the whole group $G$. Thus, all the operators in $G$ leave each of the four systems of 
imprimitivity invariant; that is, they affect the identity permutation on the set of them, which has sign equal to 1 . Consequently, $\operatorname{Ad}(G)=\{E\}$, which is false.

Corollary 7.2. If the representation $(G / H):(V / H)$ is irreducible, then the quotient $\left(V \oplus \mathbb{R}^{d}\right) / G$ is not a smooth manifold for any $d$.

This completes the proof of Theorem 1.5

Lemma 7.3. If the group $G$ is reducible over the field $\mathbb{R}$ or the group $H$ is irreducible over the field $\mathbb{C}$, then the representation $(G / H):(V / H)$ is reducible.

Proof. First suppose that $G$ is a reducible group. Then $V$ is a direct sum of nonzero $G$-invariant complex subspaces $V^{\prime}$ and $V^{\prime \prime}$ and each complex reflection in $G$ acts on one of them trivially, and on the other by a reflection. Then $V / H \cong\left(V^{\prime} / H\right) \times\left(V^{\prime \prime} / H\right)$, $V^{\prime} / H \cong V^{\prime}, V^{\prime \prime} / H \cong V^{\prime \prime}$, and the group $G / H$ acts linearly on the quotients $V^{\prime} / H$ and $V^{\prime \prime} / H$. The linear representation of the group $G / H$ in the space $V / H$ is reducible as the direct sum of its representations in the nonzero spaces $V^{\prime} / H$ and $V^{\prime \prime} / H$.

Now suppose that the group $H$ is irreducible. The classification of all the finite irreducible subgroups $H \subset \mathbf{G L}_{3}(\mathbb{C})$ generated by reflections is given in [4], and the set of degrees of homogeneous generators of the algebra of invariants $\mathbb{C}[V]^{H}$ is indicated for each of them. Thus, if the group $H$ is primitive, then the degrees of homogeneous generators of $\mathbb{C}[V]^{H}$ are pairwise distinct. On the other hand, if $H$ is imprimitive, then $H=G(p q, p, 3)$ for some $p, q \in \mathbb{N}$. The algebra $\mathbb{C}[V]^{H}$ has homogeneous generators of degrees $p q, 2 p q, 3 q$ (note that for $p \neq 3$ the numbers $p q, 2 p q, 3 q$ are pairwise distinct). In any case, among the degrees of homogeneous generators of $\mathbb{C}[V]^{H}$ at least two are distinct; therefore some coordinate line of the complex space $V / H$ is invariant under $G / H$.

This completes the proof of Theorem 1.6. the first assertion was proved at the beginning of this section and the second follows from Lemma 7.3 and Theorem 1.5 Furthermore, if the group $H$ is irreducible and does not coincide with any of the groups $G(3 q, 3,3), q \in \mathbb{N}$, then $V / G$ is a manifold, since the degrees of homogeneous generators of the algebra of invariants $\mathbb{C}[V]^{H}$ are pairwise distinct. Thus Theorem 1.7 is also proved.

We now prove Theorem 1.8 .

Suppose that the group $H$ is reducible over $\mathbb{C}$ and $G$ is irreducible over $\mathbb{R}$. Then the group $G^{0}$ acts on $V$ by scalar operators.

Suppose that $\left(V \oplus \mathbb{R}^{d}\right) / G$ is a smooth manifold for some $d \geq 0$. By Theorem 1.5, the representation $(G / H): V / H)$ is reducible, unlike the representation $G: V$; in particular, the group $H$ is nontrivial.

The representation $H: V$ cannot be isotypic; otherwise $V$ would be the direct sum of three $H$-invariant lines and the group $H$ would act on $V$ by scalar operators, but at the same time be generated by reflections and be nontrivial. Consequently (see [3, $\S 9]$ ), the representation $G: V$ has a system of imprimitivity consisting of three $H$-invariant complex lines (which can be assumed to be the coordinate lines) and is induced by a representation of a proper subgroup $\widetilde{G} \neq G, H \subset \widetilde{G} \subset G$, on the line $\mathbb{C} e_{1} \subset V$. Therefore the group $H$ consists precisely of all the diagonal operators $h$ such that $h^{q}=E$, $q>1$; that is, $H \triangleleft G^{\prime}(q, 1,3)$ is a subgroup of index 3 . The algebra of invariants $\mathbb{C}[V]^{H}$ has homogeneous generators $z_{i}^{q}$ all with the same degree $q$, and under the factorization map $V \rightarrow V / H$ given by $\left(z_{1}, z_{2}, z_{3}\right) \rightarrow\left(z_{1}^{q}, z_{2}^{q}, z_{3}^{q}\right)$ the group $G^{0}$ acts on $V / H$ by scalar operators.

The reducible representation $(G / H):(V / H)$ is induced by a one-dimensional representation of the subgroup $\widetilde{G} / H$ on the line $\mathbb{C} e_{1} \subset V / H$. According to Mackey's irreducibility criterion $([3, \S 7])$, any operator in $G^{\prime}$ that leaves all three coordinate lines of the spaces $V$ and $V / H$ invariant acts as a scalar on $V / H$. Furthermore, the group $G^{\prime}$ 
affects only even permutations on the set of coordinate lines in $V$ and $V / H$, since none of the elements of $G / H$ acts on $V / H$ by a complex reflection. We can assume that $G^{\prime}$ is generated by the subgroup $G^{0} H$ and the operator of a cyclic permutation of the coordinates, while the line $\mathbb{C} v$, where $v:=(1,1,1) \in V / H$, is invariant under $G / H$. Thus, $G^{\prime}=G^{0} G^{\prime}(q, 1,3)=G^{\prime}(q, 3) \subset G(q, 3)$.

It remains to prove that the operator $g_{(2,3)}$ belongs to $G$. The group $H$ leaves all three coordinate lines invariant; hence, using Corollary 2.8, some antilinear involution $g \in \Omega$ permutes them nontrivially, and therefore by a transposition. We can assume that $g\left(z_{1}, z_{2}, z_{3}\right)=\left(\lambda_{1} \overline{z_{1}}, \lambda \overline{z_{3}}, \lambda \overline{z_{2}}\right),|\lambda|=\left|\lambda_{1}\right|=1$. Since under the action of $g$ the vector $v=(1,1,1) \in V / H$ goes to the vector $\left(\lambda_{1}^{q}, \lambda^{q}, \lambda^{q}\right) \in V / H$, we have $\lambda_{1}^{q}=\lambda^{q}$ and $g_{(2,3)} \in g G^{0} H \subset G$.

Conversely, let $G=G(q, 3)$. The group $H$ consists exactly of all the diagonal operators $h$ such that $h^{q}=E$, and the factorization map $V \rightarrow V / H$ can be given by the formula $\left(z_{1}, z_{2}, z_{3}\right) \rightarrow\left(z_{1}^{q}, z_{2}^{q}, z_{3}^{q}\right)$. It is easy to see that the representation of the group $G$ in the space $V / H$ is reducible: the complex line spanned by the vector $(1,1,1) \in V / H$ remains invariant. It remains to prove that $\langle\Omega\rangle=G$. The antilinear involutions $g_{(2,3)}$ and $g_{(1,2)}:\left(z_{1}, z_{2}, z_{3}\right) \rightarrow\left(\overline{z_{2}}, \overline{z_{1}}, \overline{z_{3}}\right)$ belong to $G$; therefore the group $\langle\Omega\rangle$ affects various permutations on the set of three lines $\mathbb{C} e_{i} \subset V$. The subgroup of all operators of the group $G$ that leave invariant all the coordinate lines coincides with $G^{0} H$. We have $\langle\Omega\rangle \supset H$, as well as $\langle\Omega\rangle \supset G^{0}$, since $\operatorname{Ad}(\Omega) \ni-E$; therefore $\langle\Omega\rangle=G$.

Theorem 1.8 is proved.

\section{The 'SIMPLIFICATION' OF REPRESENTATIONS}

8.1. General construction. Suppose that there is an abstract group $G$ and a subgroup $H \subset G$ acting on some (abstract) set $F$. We again denote by $G / H$ the set of left cosets of $H$ in $G$. We consider the action of the group $H$ on the set $G \times F$ by the rule $h(g, f):=\left(g h^{-1}, h f\right)$ and the action of the group $G$ by left shifts of the first argument: $g^{\prime}(g, f):=\left(g^{\prime} g, f\right)$. The action of the group $G$ commutes with the action of $H$, since left and right shifts commute. Therefore $G$ acts canonically on the quotient $(G \times F) / H$, that is, on the homogeneous fibration $G_{H}^{*} F$, so that the surjective factorization map $\pi_{0}: G \times F \rightarrow G \underset{H}{*} F$ commutes with the action of $G$ on the sets $G \times F$ and $G \underset{H}{*} F$. The surjection $\pi^{\prime}: G \times F \rightarrow G / H$ given by $(g, f) \rightarrow g H$ is constant on the fibres of $\pi_{0}$ and commutes with the action of $G$ on $G \times F$ and $G / H$ by left shifts. Therefore, there exists a surjective map $\pi: G \underset{H}{*} F \rightarrow G / H$ commuting with the action of $G$ and such that $\pi^{\prime}=\pi \circ \pi_{0}$.

If $f, f^{\prime} \in F$ are distinct elements, then $\pi_{0}(e, f)$ and $\pi_{0}\left(e, f^{\prime}\right)$ are distinct elements of $G \underset{H}{*} F$; therefore we identify the element $\pi_{0}(e, f) \in G_{H}^{*} F$ (for $f \in F$ ) with $f$. Then $\pi^{-1}(e H)=\pi_{0}(H \times F)=\pi_{0}(\{e\} \times F)=F$, and since $\pi$ commutes with the action of $G$, we have $\pi^{-1}(g H)=g \pi^{-1}(e H)=g F$. Thus, $\pi$ is a fibration with fibre $F$. We consider the set $S_{H}(G, F)$ of its sections, i.e., maps $s: G / H \rightarrow G_{H}^{*} F$ such that $\pi \circ s=\operatorname{id}_{G / H}$. The group $G$ acts canonically on $S_{H}(G, F)$ by the rule $g(s):=g \circ s \circ g^{-1}$, since

$$
\pi\left(g s g^{-1}\right)=g \pi s g^{-1}=g \circ \operatorname{id}_{G / H} \circ g^{-1}=\operatorname{id}_{G / H} .
$$

If $F$ is a linear space and $H$ acts linearly on $F$, then the fibre of the fibration $\pi$ is a linear space. Therefore all the sections of the fibration $\pi$ also form a vector space $S_{H}(G, F)$ and the action $G: S_{H}(G, F)$ is linear. If in addition $\operatorname{dim} F<\infty$ and $|G / H|<\infty$, then the space $S_{H}(G, F)$ is finite-dimensional.

Below we describe a method for reducing the original linear representation $G: V$ to representations in spaces of lower dimension. Namely, suppose that the space $V$ 
contains a direct sum $S$ of pairwise orthogonal subspaces permuted by the group $G$ so that $G$ acts transitively on the (finite) set $M$ of these subspaces. Then the product of all subgroups of the form $G[W] \subset G$ (for $W \in M$ ) is direct. We set $T:=S^{\perp} \subset V$. Clearly, $G S=S, G T=T$, and the set of subspaces $M \cup\{T\}$ is a system of imprimitivity of the representation $G: V$.

We now fix some subspace $W \in M$. Let $G(W):=\{g \in G: g W=W\} \subset G$ be the stabilizer of an element $W \in M$ in the group $G$; this is a Lie subgroup of finite index $|G / G(W)|=|M|$, so $G^{0} \subset G(W)$. Obviously, $G[W] \subset G(W)$; moreover, $G[W] \triangleleft G(W)$.

Consider some subgroup $H \subset G[W]$ that is normal in $G(W)$. It acts trivially on $W^{\perp}$ and therefore embeds into $\mathbf{O}(W)$. For any $g \in G$ we have $g H g^{-1} \subset G[g W]$. Furthermore, if $g_{1} W=g_{2} W, g_{i} \in G$, then $g_{0}:=g_{1}^{-1} g_{2} \in G(W)$ and $g_{0} H g_{0}^{-1}=H$, whence $g_{2} \mathrm{Hg}_{2}^{-1}=g_{1} \mathrm{Hg}_{1}^{-1}$. Thus, in the group $\mathrm{G}$, distinct subgroups of the form $\mathrm{gHg}^{-1}$ are contained in distinct groups of the form $G[g W], g \in G$. Therefore, only finitely many among the subgroups $g H^{-1}$ are distinct (at most $|M|$ ) and their product $H^{\prime}$ is direct. Subgroups of the form $\mathrm{gHg}^{-1}$ are permuted under conjugation by elements of $G$; consequently, $H^{\prime} \triangleleft G$.

Proposition 8.1. Suppose that the quotient $W / H$ is diffeomorphic to a vector space $W^{\prime}$ on which the group $G(W)$ acts linearly. Then the quotient $V / H^{\prime}$ is diffeomorphic to some vector space $V^{\prime}$ on which $G$ acts linearly.

Proof. The subgroup $G(W) \subset G$ of finite index $|M|$ acts linearly in the space $W^{\prime}$; therefore the set $S^{\prime}:=S_{G(W)}\left(G, W^{\prime}\right)$ is a finite-dimensional vector space and $G$ acts on it linearly. By hypothesis, the quotient $S / H^{\prime}$ is diffeomorphic to the finite-dimensional vector space $S^{\prime}$ and the canonical action of the group $G$ on this quotient is linear. Also, since $H^{\prime}$ acts on $T$ trivially, the quotient $V / H^{\prime}$ is diffeomorphic to the finite-dimensional space $V^{\prime}:=S^{\prime} \oplus T$ on which $G$ acts linearly.

Let $G^{\prime} \subset \mathbf{G L}\left(V^{\prime}\right)$ be the image of the group $G$ under the linear representation $G: V^{\prime}$ obtained (under the hypotheses of Proposition 8.1). Then the quotient $V / G$ is a (smooth) manifold if and only if $V^{\prime} / G$ is a (smooth) manifold. Thus the question of whether the quotient of the representation $G: V$ is a (smooth) manifold reduces to a similar question for the faithful linear representation $G^{\prime}: V^{\prime}$. If in addition $\operatorname{dim} H>0$, then $\operatorname{dim} H^{\prime}>0$ and $\operatorname{dim} V^{\prime}=\operatorname{dim}\left(V / H^{\prime}\right)<\operatorname{dim} V$; that is, we have succeeded in reducing the original representation to a representation of strictly lower dimension.

8.2. Passing to a 2-stable set of weights. We return to the action of the group $G$ in the space $V$ and to weights $\lambda: G^{0} \rightarrow \mathbb{T}$ and $\lambda: \mathfrak{g} \rightarrow \mathbb{R}$ corresponding to a decomposition into irreducible components (accounting for multiplicities). The set of these weights in the space $\mathfrak{g}$ is again denoted by $P$. As we mentioned, the set $P$ is independent of the choice of decomposition (up to changing the sign of some of its elements). We assume that $P$ is 1-stable; otherwise $V / G$ is not a manifold according to Proposition 2.2. Suppose that $P$ is not a 2-stable set. Our aim is to reduce the study of the quotient of the original representation to the study of the quotient of another faithful representation $G^{\prime}: V^{\prime}$ such that $\operatorname{dim} V^{\prime}<\operatorname{dim} V$. The main tool we shall use is the method for passing to another representation, which we described in Subsection 8.1. To prove that some subspace $W$ and some subgroup $H \subset G[W]$ that is normal in $G(W)$ satisfy the hypotheses of Proposition 8.1 we shall use the examples in Subsection 3.2

Any weight $\lambda \in \mathfrak{g}$ can be replaced by its opposite; therefore we can make sure that all the coefficients of the form $c_{\lambda}, \lambda \in P$, are positive (see \$4).

Every operator $\operatorname{Ad}(g), g \in G$, leaves invariant, up to sign, the set $P$ and permutes its equivalence classes. 
Since the set $P$ is not 2 -stable, there exists an equivalence class $N$ in it with more than one element. This class does not contain trivial weights and, in addition, together with any of its weights, it also includes all its copies in $P$. We set $W:=V_{N}$. Any pair of images of the set $N$ under the action of all the operators $\operatorname{Ad}(g), g \in G$ either coincide or are disjoint (up to sign). Therefore any pair of subspaces $g W, g \in G$, either coincide or are orthogonal. Their sum is direct, and they are permuted transitively under the action of $G$. Let $H:=G[W] \cap G^{0} \subset G[W]$. This subgroup is normal in $G(W)$, since $G[W] \triangleleft G(W)$ and $G^{0} \triangleleft G$.

Proposition 8.2. For this choice of the subspace $W$ and subgroup $H \subset G[W]$, the quotient $W / H$ is diffeomorphic to some vector space $W^{\prime}$ on which the group $G(W)$ acts linearly.

Proof. It is easy to see that the subgroup $H=G[W] \cap G^{0}$ coincides with $\bigcap_{\lambda \in P \backslash N} \operatorname{Ker} \lambda$ (where the $\lambda: G^{0} \rightarrow \mathbb{T}$ are weights), and the subalgebra $\mathfrak{h}:=$ Lie $H=\mathfrak{g}[W]$ with $\bigcap_{\lambda \in P \backslash N} \operatorname{Ker} \lambda$ (where the $\lambda$ are weights in $\mathfrak{g}$ ).

The group of restrictions of operators in $G^{0}$ to the subspace $W$ acts on each of its irreducible components by multiplication by a complex number; therefore it naturally embeds into the torus $\mathbb{T}^{\|N\|}$ so that the image under the embedding is just $\varphi_{N}\left(G^{0}\right)$. As for the group $H$, it also embeds into $\mathbb{T}^{\|N\|}$; the image under the embedding is $\varphi_{N}(H)$.

We restrict the homomorphism $\varphi_{N}$ to the Lie subgroup $H \subset G^{0}$. If some character

$$
\chi: T^{\|N\|} \rightarrow \mathbb{T}, z \rightarrow \prod_{\lambda \in N} z_{\lambda}^{d_{\lambda}}, d_{\lambda} \in \mathbb{Z}
$$

is trivial on the whole subgroup $\varphi_{N}(H) \subset \mathbb{T}^{\|N\|}$, then the linear function

$$
d \chi: \mathbb{R}^{\|N\|} \rightarrow \mathbb{R}, x \rightarrow \sum_{\lambda \in N} d_{\lambda} x_{\lambda}
$$

is trivial on the algebra $\operatorname{Lie} \varphi_{N}(H)=d \varphi_{N}(\mathfrak{h}) \subset \mathbb{R}^{\|N\|}$. The latter is equivalent to the fact that the function $\sum_{\lambda \in N} d_{\lambda} \lambda$ is equal to zero on the subspace $\mathfrak{h}=\bigcap_{\lambda \in P \backslash N} \operatorname{Ker} \lambda$ or, equivalently, it belongs to $\langle P \backslash N\rangle$. Since $N$ is an equivalence class, up to proportionality exactly one of these linear combinations is nontrivial, and its coefficients $c_{\lambda}(\lambda \in N)$ are positive. Therefore $\operatorname{Lie} \varphi_{N}(H)$ is a hyperplane in $\mathbb{R}^{\|N\|}$, while the subgroup $\varphi_{N}(H) \subset$ $\mathbb{T}^{\|N\|}$ has codimension 1 and is defined by a single nontrivial character with exponents proportional to the $c_{\lambda}$ and therefore they are nonzero and have the same sign (we can assume they are positive integers).

The space $W$ is a direct sum of $\|N\|$ pairwise orthogonal irreducible components; each of them is a one-dimensional complex space on which the group $G^{0}$ (and therefore also $H$ ) acts by multiplication by complex numbers. The unique nontrivial linear combination of the weights $\lambda \in N$, up to proportionality, contained in the linear hull of the other weights in $P$ has coefficients $c_{\lambda}>0$. As already mentioned, $H$ is a subgroup of the $\|N\|$-dimensional torus defined by a single character with positive integer exponents $a_{\lambda}$ which are proportional to the $c_{\lambda}$ :

$$
\varphi_{N}(H)=\left\{z \in \mathbb{T}^{\|N\|}: \prod_{\lambda \in N} z_{\lambda}^{a_{\lambda}}=1\right\} \subset \mathbb{T}^{\|N\|} ;
$$

in particular, $\operatorname{dim} H=\|N\|-1>0$. For such a group $H$, the quotient $W / H$ is diffeomorphic to a vector space $W^{\prime}$ of dimension $\|N\|+1$ by Theorem 3.4. Furthermore, the restriction to $W$ of any operator in $G(W)$ is an orthogonal operator in $W$ contained in the normalizer of the group $H$; consequently, it acts linearly on the quotient $W / H$ (again by Theorem 3.4). 
According to Proposition 8.1 we can reduce the original action $G: V$ to another faithful representation $G^{\prime}: V^{\prime}$ such that $V / G$ is a (smooth) manifold if and only if $V^{\prime} / G^{\prime}$ is a (smooth) manifold. Next, $\operatorname{dim} H>0$, whence $\operatorname{dim} V^{\prime}<\operatorname{dim} V$. We continue this procedure until we obtain a 2 -stable set of weights. Since the dimension of the space decreases strictly at each step, sooner or later the process will terminate and therefore the set of weights will become 2 -stable.

\section{REFERENCES}

1. M. A. Mikhălova, On the quotient space modulo the action of a finite group generated by pseudoreflections, Izv. Akad. Nauk SSSR Ser. Mat. 48, no. 1 (1984), 104-126; English transl. in Math. USSR-Izv. 24 (1985), 99-119. MR733360 (85k:20127)

2. G. E. Bredon, Introduction to compact transformation groups, Pure and Applied Mathematics, Vol. 46, Academic Press, New York-London, 1972. MR0413144 (54:1265)

3. J.-P. Serre, Représentations linéaires des groupes finis, Hermann, Paris, 1978. MR543841|(80f:20001)

4. G. C. Shephard and J. A. Todd, Finite unitary reflection groups, Canadian J. Math. 6 (1954), 274304. MR0059914 (15:600b)

5. H. F. Blichfeldt, Finite collineation groups, Univ. of Chicago Press, 1917.

Moscow State University, Moscow, Russia

Translated by E. KHUKHRO 